\title{
Parameter Sweep Experiments on a Spectrum of Cyclones with Diabatic and Baroclinic Processes
}

\author{
WATARU YANASE \\ Meteorological Research Institute, Japan Meteorological Agency, Tsukuba, Japan \\ HIROSHI NIINO \\ Atmosphere and Ocean Research Institute, The University of Tokyo, Kashiwa, Japan
}

(Manuscript received 6 August 2018, in final form 10 April 2019)

\begin{abstract}
A wide range of environments that prevail over the globe generate various types of cyclones such as tropical, extratropical, and hybrid cyclones. In this paper, idealized numerical experiments are used to explore a spectrum of cyclones ranging from the diabatic type to the baroclinic type in a parameter space consisting of three environmental factors: temperature, vertical shear, and planetary vorticity. The experiments reproduce not only typical dynamics of tropical and extratropical cyclones but also their modified dynamics, which are consistent with theoretical studies; tropical cyclones are suppressed by vertical shear, while extratropical cyclones are intensified by condensational heating. The experiments also reproduce hybrid cyclones in environments with high temperature and large baroclinicity. The hybrid cyclones show multiscale dynamics in which synoptic-scale baroclinic systems spawn smaller-scale tropical cyclone-like convective cores. The spectrum of cyclones is found to be nonmonotonic in the parameter space because of a two-sided effect of the vertical shear: moderate shear weakens a tropical cyclone by tilting the small-scale vortex to the downshear, while strong shear develops a large-scale vortex of an extratropical cyclone or a hybrid cyclone through warm-air advection from the south. The indices based on the energetics and the symmetric and asymmetric structures overview the different types of cyclones in the parameter space. These parameter sweep experiments provide useful information on what environment is favorable for cyclones, particularly for intermediate environments where cyclone mechanisms are yet to be fully defined.
\end{abstract}

\section{Introduction}

Observations and numerical simulations show that various types of synoptic-scale cyclones including tropical cyclones (TCs), extratropical cyclones (ECs), and other types of cyclones develop over the globe. The different types of cyclones are caused by different mechanisms depending on their environments. A major energy source of a TC is condensational heating of water vapor over warm oceans at low latitudes (Charney and Eliassen 1964; Ooyama 1969; Emanuel 1986; Montgomery and Smith 2014). On the other hand, that of an EC is baroclinic processes in an environment with large baroclinicity (large vertical shear and large planetary vorticity) at midlatitudes (Charney 1947; Eady 1949; Hoskins 1976; Catto 2016).

While a typical TC and a typical EC are entirely different in structures and mechanisms, there are intermediate

Corresponding author: Wataru Yanase, wtryanase@gmail.com or hybrid types of cyclones (HCs), which include subtropical cyclones and cyclones during extratropical transition and tropical transition (Hart 2003; da Rocha et al. 2019). Subtropical cyclones extract available potential energy through baroclinic processes as ECs do, but they also receive some or most of their energy from condensational heating as TCs do (American Meteorological Society 2019). Guishard et al. (2009) showed that subtropical cyclones over the North Atlantic Ocean are most active in September and October when the region of high sea surface temperature (SST) is overlapped with the region of large baroclinicity. Extratropical transition is a familiar process by which a TC transforms into an EC within a baroclinic environment at midlatitudes (Jones et al. 2003; Kitabatake 2011; Evans et al. 2017), while tropical transition is also an important process by which an EC or HC transforms into a TC (Davis and Bosart 2004; Mauk and Hobgood 2012; McTaggart-Cowan et al. 2013; Bentley et al. 2016). 
It is sometimes not easy to classify HCs accurately, because some HCs are closer to TCs, while others are closer to ECs. Thus, synoptic-scale cyclones encompass a broad spectrum of cyclones between typical TCs and typical ECs.

Climatologically, cyclones tend to develop in their favorable environments. TCs usually develop over warm oceans in the tropics (Gray 1975), and ECs in the regions with large baroclinicity in the extratropics (Hoskins and Valdes 1990). HCs often occur in the intermediate environment between the tropics and the extratropics (Guishard et al. 2009; Yanase et al. 2014). Cyclones may experience drastically changing environments during their life cycle; for example, an extratropical transitioning TC moves from tropical environments to extratropical environments (Hart and Evans 2001; Evans et al. 2017). In addition to the effect of environments, cyclones are also affected by transient external forcing such as upper-tropospheric troughs (Deveson and Browning 2002; Bentley et al. 2017; Fischer et al. 2017). In the real atmosphere, these processes influence cyclones simultaneously and intricately. To understand the climatology of cyclones, it is necessary to elucidate the individual processes. The present study will address an issue of what kind of cyclones develop in timeindependent environments in the absence of transient external forcing.

The development of cyclones in simplified environments has been explored by a number of theoretical studies. Early theoretical studies on ECs have clarified the adiabatic dynamics of baroclinic waves using linear models (Eady 1949; Charney 1947) and three-dimensional atmospheric models (Mudrick 1974; Takayabu 1986). Later, the dynamics of baroclinic waves modified by diabatic heating has also been studied using linear models with parameterization of diabatic heating and three-dimensional full-physics models. In general, baroclinic waves become more intense when diabatic heating are incorporated (Mak 1982; Wang and Barcilon 1986; Lambaerts et al. 2012; Booth et al. 2013). Lapeyre and Held (2004) showed that large condensational heating causes a vortex dominant regime that has no analog in the dry model.

Theoretical studies on a typical TC have clarified axisymmetric dynamics using simplified axisymmetric models (Charney and Eliassen 1964; Ooyama 1969; Emanuel and Rotunno 1989) and multiscale dynamics using three-dimensional high-resolution models with full physics (Hendricks et al. 2004). The dynamics of TCs modified by vertical shear have also been studied by three-dimensional full-physics models (Frank and Ritchie 2001; Riemer et al. 2010; Finocchio et al. 2016). In general, the vertical shear suppresses the development of TCs and causes asymmetric structure where an updraft tends to occur on the downshear side of the cyclone center rather than the upshear side. According to Jones (1995), an initially upright vortex in the vertical shear of zonal wind can adiabatically develop an updraft on the downshear side by two different mechanisms, which will be referred to as the "warmair advection" and "downshear tilt" mechanisms in the present study. The warm-air-advection mechanism explains the updraft through the cyclonic circulation along the meridionally sloped isentropic surface (i.e., meridional temperature advection at a constant altitude) within the meridional temperature gradient balanced with vertical shear. The downshear-tilt mechanism associates the updraft with the adiabatic cooling that satisfies the thermal wind balance with the tilted vortex. While both mechanisms explain the updraft on the downshear side of the cyclone, they are characterized by different temperature anomalies.

In contrast to TCs and ECs, which are located at the two extreme ends of the cyclone spectrum, it is difficult to determine a typical environment for $\mathrm{HCs}$, which are located in a broad range of the spectrum. As for subtropical cyclones, there have been only a few idealized experiments. Davis (2010) conducted the first idealized experiments of subtropical cyclones, which develop from deep moist convection organized by synoptic-scale disturbances in an environment with moderate vertical shear. Yanase and Niino (2018, hereafter YN18) conducted idealized experiments using the environmental fields over the subtropical North Atlantic Ocean in autumn, and reproduced the multiscale structure of HCs, which is similar to the composite structure of subtropical cyclones in the real atmosphere (Evans and Guishard 2009).

To examine the influence of environmental fields on different types of cyclones, YN18 also compared the experiments using the environmental fields in the tropics, subtropics, and extratropics. These experiments demonstrated that the environmental fields can reproduce the contrast of TCs, ECs, and HCs over the North Atlantic Ocean qualitatively. While this study examined a wide range of environmental factors including temperature, vertical shear, and the Coriolis parameter, the climatological fields do not cover all the possible combinations of the factors. In addition, it is difficult to elucidate the influence of individual environmental factors in the real atmosphere, because all the factors change simultaneously.

To understand the broad spectrum of cyclones from a typical TC to a typical EC more comprehensively, the present study conducts parameter sweep experiments, which explore the parameter space of environmental factors systematically. We consider that one of the 
minimal set of environmental factors that effectively changes diabatic and baroclinic processes would be SST $T$, vertical shear $U$, and planetary vorticity $F$; diabatic process would become more dominant in higher temperature environment, because the Clausius-Clapeyron equation gives more water vapor; baroclinic process would become more dominant in stronger vertical shear and larger planetary vorticity. In this $T-U-F$ parameter space of the environmental fields, we can expect that a typical TC will develop in the environment with large $T$, small $U$, and small $F$, whereas a typical EC will develop in the environment with small $T$, large $U$, and large $F$. Here, two questions arise as to cyclones outside of these typical environments for TCs and ECs. 1) In which region of the parameter space do cyclones develop? In particular, it is not straightforward whether cyclones in the environment with large $T$, large $U$, and large $F$ are intense or not; an EC may be intensified by large condensational heating in large- $T$ environment, whereas a TC may be weakened in large- $U$ environment. 2) What are the structures and dynamics of cyclones other than typical TCs and ECs? Asymmetric structures of cyclones may be caused by the aforementioned warm-air-advection or downshear-tilt mechanisms depending on the environments. In addition, multiscale structures and dynamics of cyclones may be simulated by a three-dimensional full-physics model, which is an advantage over linear models and axisymmetric models.

The remaining sections are organized as follows. Section 2 describes experimental design including the specification of a nonhydrostatic model and environmental fields. Section 3 describes the results of representative experiments and several other experiments in the $T-U-F$ parameter space. Section 4 compares the axisymmetric and asymmetric structures between different types of cyclones. Section 5 discusses the result of the present study in the context of theoretical, numerical, and observational studies. Finally, section 6 summarizes the spectrum of cyclones in the $T-U-F$ parameter space.

\section{Methodology}

In contrast to the simplicity of our purpose, the experiments have to be designed carefully to deal with various types of cyclones. The methodology in the present study is almost the same as that in YN18 except for environmental fields (section $2 \mathrm{~b}$ ) and analysis of cyclones (section 2d).

\section{a. A numerical model}

Multiscale dynamics of various cyclones are simulated by three-dimensional numerical experiments using the
Japan Meteorology Agency Nonhydrostatic Model (JMA-NHM; Saito et al. 2006). The cloud processes are calculated by a bulk-type microphysics scheme that considers water vapor, cloud water, cloud ice, rain, snow, and graupel (Lin et al. 1983; Murakami 1990) and by a convective parameterization scheme (Kain and Fritsch 1990). The planetary boundary layer is represented by the level-2.5 closure of Mellor-YamadaNakanishi-Niino turbulence scheme (Nakanishi and Niino 2004) together with the surface-layer scheme developed by Beljaars (1995). For simplicity, radiative processes and ocean feedback are not considered.

The model domain is 8000,3000 , and $24.52 \mathrm{~km}$ in the zonal, meridional, and vertical directions, respectively. The zonal boundary conditions are cyclic, whereas the meridional boundaries are free slip and adiabatic walls. The horizontal grid spacing is $10 \mathrm{~km}$ throughout the domain, and the vertical grid spacing is increased linearly from $40 \mathrm{~m}$ at the lowest level to $1360 \mathrm{~m}$ at the highest level of the domain.

\section{b. Environmental fields}

The environmental fields are constructed based on the climatological fields in the North Atlantic Ocean during the autumn season (September-November), because it is known that various types of cyclones can be simulated using the environmental fields in this region (YN18). The climatological fields for 30 years (1982-2011) are obtained from the JRA-25 atmospheric dataset (Onogi et al. 2007) and NOAA's Optimum Interpolation SST product (Reynolds et al. 2002). The atmospheric profiles and SST are averaged within the area of $10^{\circ}-50^{\circ} \mathrm{N}$, $40^{\circ}-60^{\circ} \mathrm{W}$ to make "reference profiles," which are to be further modified to construct the profiles for individual experiments. The reference profiles for potential temperature, relative humidity, and zonal wind are presented in Fig. 1, while the reference SST is $23.6^{\circ} \mathrm{C}$. The zonal wind below $1-\mathrm{km}$ height is prescribed by extrapolating the average gradient between 1 - and $1.5-\mathrm{km}$ heights to remove small-scale fluctuations in the boundary layer. The zonal wind is further shifted by a constant value at all heights in order to make the wind speed at the sea level vanish. This minimizes the influence of surface friction and surface heat fluxes associated with environmental zonal flow at the sea level.

The environment for each experiment is determined by three parameters: temperature, vertical shear, and planetary vorticity (Table 1$)$. The temperature parameter $T$ represents the SST, which is adjusted by adding a constant value to the reference SST as well as to the reference profile of atmospheric temperature. Therefore, the dry static stability in the atmosphere and temperature difference between the atmosphere 
(a)

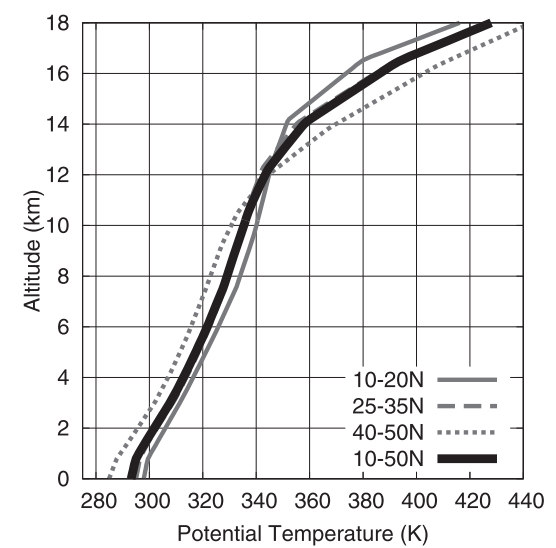

(b)

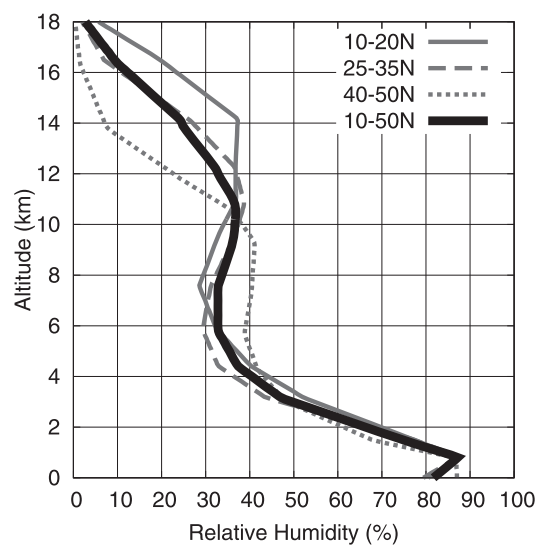

(c)

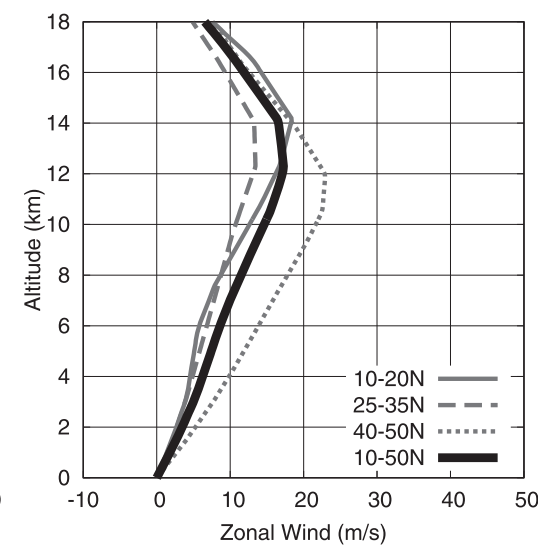

FIG. 1. Vertical profiles of environmental fields over the North Atlantic Ocean (zonal average over $40^{\circ}-60^{\circ} \mathrm{W}$ ) in autumn: (a) potential temperature $(\mathrm{K})$, (b) relative humidity $(\%)$, and (c) zonal wind $\left(\mathrm{m} \mathrm{s}^{-1}\right)$. The thick black curves are reference profiles (meridional average over $\left.10^{\circ}-50^{\circ} \mathrm{N}\right)$, which are used for the present idealized experiments. The thin gray curves are profiles in the different latitudes just for information: meridional averages over $10^{\circ}-20^{\circ}$ (thin black solid), $25^{\circ}-35^{\circ}$ (dashed), and $40^{\circ}-50^{\circ} \mathrm{N}$ (dotted).

and ocean are nearly the same for all the experiments. The vertical shear parameter $U$ represents the difference of the zonal wind between 200 and $850 \mathrm{hPa}$. The vertical profile of zonal wind is adjusted by multiplying the reference profile by a constant value. The planetary vorticity parameter $F$ represents the Coriolis parameter normalized by that at $90^{\circ} \mathrm{N}$ (i.e., sine of the latitude). Hereafter, the experiments will be referred to as $\mathrm{T} x x \mathrm{U} y y \mathrm{~F} z z$, where $x x, y y$, and $z z$ are digits denoting temperature, vertical shear, and planetary vorticity, respectively; for example, in the T26U20F50 experiment, the SST is $26^{\circ} \mathrm{C}$, the vertical shear is $20 \mathrm{~m} \mathrm{~s}^{-1}$, and the Coriolis parameter is $50 \%$ (the value at $30^{\circ} \mathrm{N}$ ). The profile of relative humidity is fixed for all the experiments.

Three-dimensional environmental fields for the experiments are constructed by combining the abovementioned vertical profiles with the following simple horizontal distributions. All of the environmental fields are uniform in the zonal direction. The Coriolis parameter is constant (the $f$-plane approximation). Zonal wind, relative humidity, and surface temperature difference between the atmosphere and ocean are uniform in the meridional direction except for some modification to be described later. The vertical shear of the zonal wind should be in thermal wind balance with the meridional gradient of potential temperature according to the Coriolis parameter. Thus, meridional gradient of potential temperature is superposed on the horizontally uniform field based on the given vertical profile (Fig. 2b). Since the warmer region near the southern boundary turned out to cause undesirable convective disturbances, the environmental fields within $500 \mathrm{~km}$ from the northern and southern boundaries (boundary zones) are modified to suppress convection as follows. The zonal wind in the boundary zones is reduced by multiplying a weighting function as shown in Fig. 2a, which results in a weaker meridional temperature gradient. SST and the mixing ratio of water vapor in the boundary zones are kept smaller than the maximum values in the rest of the domain (the central zone). Furthermore, Rayleigh damping are also applied to the wind, temperature, and humidity fields in the boundary zones. These experimental designs effectively suppress convective disturbances in the boundary zones.

\section{c. Time integration}

The initial condition is constructed by superposing an axisymmetric vortex, which is located at the center of the model domain, on the aforementioned environmental fields. The structure of the initial vortex is designed as simply as possible so that cyclones evolve into different structures depending on the environment. The tangential wind of the vortex is given by

$$
V=V_{\max } \frac{2 r / r_{\max }}{1+\left(r / r_{\max }\right)^{2}}\left(1-\frac{z}{z_{t}}\right)
$$

TABLE 1. Three parameters of the environment.

\begin{tabular}{lc}
\hline \multicolumn{1}{c}{ Parameter } & Definition \\
\hline Temperature $T$ & Sea surface temperature $\left({ }^{\circ} \mathrm{C}\right)$ \\
Vertical shear $U$ & Vertical shear of zonal wind \\
& between 850 and $200 \mathrm{hPa}\left(\mathrm{m} \mathrm{s}^{-1}\right)$ \\
Planetary vorticity $F$ & Coriolis parameter normalized by \\
& the magnitude at $90^{\circ} \mathrm{N}(\%)$ \\
\hline
\end{tabular}




\section{(a) U-weight}

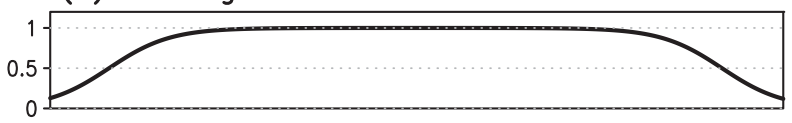

(b) Potential Temperature and Zonal Wind

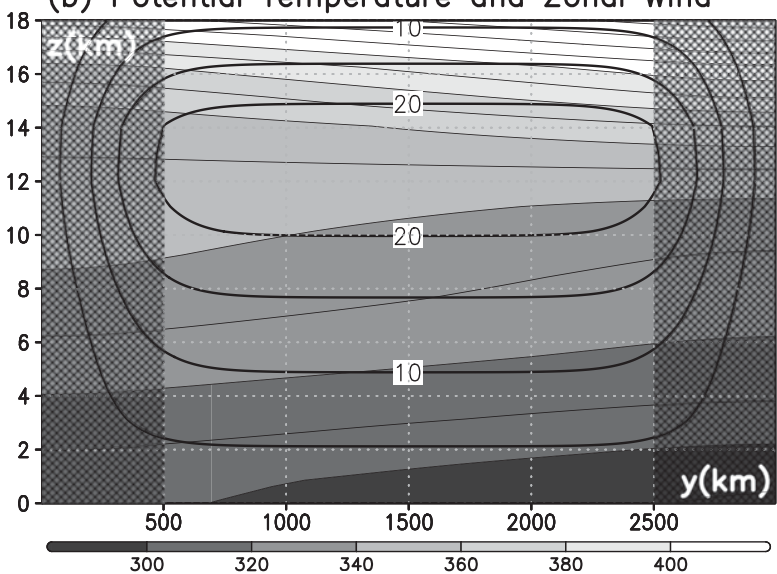

FIG. 2. Meridional and vertical structure of the environment. (a) Meridional distribution of the weighting function for zonal wind $W_{u}$. (b) Meridional and vertical distribution of potential temperature (shading and thin contours at $10-\mathrm{K}$ interval) and zonal wind (thick contours at $5 \mathrm{~m} \mathrm{~s}^{-1}$ interval). The black hatching indicates the southern and northern boundary zones. This example uses the vertical profiles of zonal wind and potential temperature for T26U20F50.

for the height of $z \leq z_{t}$, where $V_{\max }=10 \mathrm{~m} \mathrm{~s}^{-1}, r_{\max }=$ $250 \mathrm{~km}$, and $z_{t}=10 \mathrm{~km}$. The vortex has a temperature anomaly that satisfies the thermal wind balance (the hydrostatic and gradient wind balance) with the tangential wind.

During the time integration of $1200 \mathrm{~h}$, the environmental field would be modified by diffusion and disturbances, which would make the results difficult to interpret. Therefore, the environmental fields are maintained using a spectral nudging with an $e$-folding time of $10 \mathrm{~h}$, which only operates on the zonally averaged fields. Because we assume the time-independent environments, tropical and extratropical transition is outside the scope of the present study.

\section{d. Analysis of cyclones}

The model outputs on the original grid of $10 \mathrm{~km}$ (referred to as "raw fields") resolve convective systems of $10-100 \mathrm{~km}$, which are too noisy to analyze synoptic-scale cyclones. Therefore, we also utilize "filtered fields," in which wavelength less than $500 \mathrm{~km}$ are filtered out.

Because of the long integration time and the large zonal domain, an experiment may produce more than one cyclone, which develop from the initial vortex or form spontaneously. The present study detects all the cyclones in the following manner, and picks out the most intense cyclone for analysis in each experiment.
The centers of cyclones are defined by minima of sea level pressure (SLP) anomaly, where the anomaly is defined by the deviation from the zonal mean. Tracks of the cyclones are constructed by connecting nearest cyclones between consecutive time steps, where we assume simple cyclone motion based on the environmental zonal flow averaged between 0 - and $10-\mathrm{km}$ heights. The first guess of tracking uses the filtered field of SLP, which can reduce fluctuation and miscalculation of tracks due to small-scale convective systems. Then, the final location and intensity $P_{\min }$ of the cyclone is determined based on the raw fields of SLP anomaly around the first guess of the cyclone center.

We objectively define a "development stage" of a cyclone, which is used for indices to be described later. To remove short time fluctuations, 10-hourly output of $P_{\min }$ is smoothed using a 100 -h running mean. The end of the development stage (referred to as "mature time") is determined by the time of the largest $-P_{\min }(t) P_{\mathrm{gr}}(t)^{\alpha}$, where $-P_{\min }(t)$ is the intensity, $P_{\mathrm{gr}}(t)$ $\left\{\equiv-\left[P_{\min }(t)-P_{\min }(t-\Delta t)\right] / \Delta t ; \Delta t\right.$ is $\left.100 \mathrm{~h}\right\}$ is the growth rate, and $\alpha$ is a weighting factor ( 0.5 is used). The start of the development stage is defined by the time when $-\left[P_{\min }(t+0.5 \Delta t)-P_{\min }(t-0.5 \Delta t)\right] / \Delta t$ exceeds the threshold $\left(0.1 P_{\mathrm{gr}}\right.$ at the mature time $)$. This objective identification captures the development stages of cyclones successfully as shown later.

Some cyclones develop far south from the center of the meridional domain even though the disturbances are suppressed near the southern boundary zone as mentioned in section $2 \mathrm{~b}$. Since it is hard to say that such cyclones do develop within the specified environment, the present study do not deal with the cyclones whose average locations during the development stages are farther than $400 \mathrm{~km}$ from the center of the meridional domain.

\section{e. Energy budget analysis}

The development mechanism of cyclones is assessed by the budget of eddy available potential energy (EPE). The energy budget analysis in a Cartesian coordinate $(x, y, z)$ system is calculated based on the anelastic approximation using the filtered data. The EPE is defined as

$$
\mathrm{Pe} \equiv \frac{\alpha \overline{\theta^{12}}}{2}
$$

where $\theta$ is the potential temperature and

$$
\alpha \equiv \frac{\rho_{0} g}{\theta_{0}}\left(\frac{\partial \bar{\theta}}{\partial z}\right)^{-1}
$$

( $\rho_{0}$ and $\theta_{0}$ are the reference density and potential temperature, respectively, which are functions of height 
only). The overbar and prime denote zonal mean and deviation, respectively.

The equation for the EPE budget is

$$
\frac{\partial \mathrm{Pe}}{\partial t}=-\alpha \frac{\partial \bar{\theta}}{\partial y} \overline{v^{\prime} \theta^{\prime}}+\frac{\alpha \theta_{0}}{c_{p} T_{0}} \overline{q^{\prime} \theta^{\prime}}-\frac{\rho_{0} g}{\theta_{0}} \overline{w^{\prime} \theta^{\prime}}+\text { Res },
$$

where $v$ is the meridional velocity, $w$ is the vertical velocity, $q$ is the condensational heating, $c_{p}$ is the specific heat capacity, $T_{0}$ is the reference temperature, $g$ is the gravitational acceleration, and Res is the residual terms. On the right-hand side, the first term is the conversion from mean available potential energy to EPE (referred to as "baroclinic term"), the second is the generation of EPE by condensational heating (referred to as "diabatic term"), and the third is the conversion from eddy kinetic energy to EPE. The present paper assesses the baroclinic and diabatic terms.

\section{Results}

\section{a. Representative experiments}

Before exploring the entire $T-U-F$ parameter space, this subsection confirms the basic structure and dynamics of different types of cyclones. The representative experiments for a TC (T28U00F25), EC (T14U20F75), and HC (T26U20F50) are referred to as ReT, ReE, and $\mathrm{ReH}$, respectively (Table 2). As the basic characteristics of the TC and EC are similar to those in YN18 and other studies, we will describes the structures of the cyclones with an emphasis on the HC.

\section{1) Time evolution of Central pressure}

The time evolution of $P_{\min }$ for the representative experiments are shown in Fig. 3a. As has been mentioned in section $2 \mathrm{~d}$, we examine the 100 -h running mean of $P_{\text {min }}$ (thick curves) to remove short time fluctuation of the raw values (thin curves). In ReT, a cyclone develops rapidly, reaches $P_{\min }$ of $-40 \mathrm{hPa}$ at its mature time $(160 \mathrm{~h})$, and then continues to develop slowly. The development stage is reasonably identified by the objective method in section $2 \mathrm{~d}$ (the period between two red open circles in the figure). In ReE, a cyclone develops more slowly than that in $\mathrm{ReT}$, and reaches $P_{\min }$ of $-19 \mathrm{hPa}$ at the mature time $(380 \mathrm{~h})$. In $\mathrm{ReH}$, a cyclone develops for much longer time, and finally reaches $P_{\min }$ of $-47 \mathrm{hPa}$ at the mature time (710 h).

\section{2) HORIZONTAL AND VERTICAL STRUCTURES}

The SLP and cloud patterns for the representative experiments are shown in Fig. 4. In ReT (Figs. 4a,b), the
TABLE 2. Main experiments.

\begin{tabular}{ll}
\hline \multicolumn{1}{c}{ Name } & \multicolumn{1}{c}{ Description (interpretation) } \\
\hline T28U00F25 (ReT) & Representative experiment for a TC \\
T26U20F50 (ReH) & Representative experiment for an HC \\
T14U20F75 (ReE) & Representative experiment for an EC \\
T26U20F75 & Extreme experiment for an HC \\
T26U10F40 $(\mathrm{ShM})$ & Moderate-vertical-shear experiment \\
T26U25F40 $(\mathrm{ShS})$ & Strong-vertical-shear experiment \\
\hline
\end{tabular}

cyclone has a small axisymmetric structure and a cloudfree eye surrounded by eyewall convection, which is similar to a typical TC. In ReE (Fig. 4c), the cyclone shows a large comma-shaped cloud, which resembles an EC. In ReH (Figs. 4e-1), the structure of the cyclone changes through its life cycle. During the early stage around $300 \mathrm{~h}$ (Fig. 4e), the cyclone is characterized by a weak large-scale (larger than $1000 \mathrm{~km}$ ) SLP pattern with widespread clouds in the eastern part, which is somewhat similar to an EC. Between 400 and $600 \mathrm{~h}$ (Figs. 4f-h), small-scale (several hundred kilometers) convective systems appear in the western half of the large-scale system. At $650 \mathrm{~h}$ (Fig. 4i), deepening of the low pressure system is associated with an intense smallscale convective system (referred to as a "convective core") in the northwestern part of the large-scale system. At $700 \mathrm{~h}$ (Fig. 4j), the convective core moved to the southwestern part of the large-scale system, whereas a short-lived convective system is associated with a secondary pressure minimum in the northwestern part. After $700 \mathrm{~h}$ (Figs. 4j-1), the convective core becomes similar to a TC with a cloud-free eye, while the large-scale low pressure system still has widespread clouds in the eastern part. Throughout the life cycle of this HC, convective systems including the convective core tend to develop in the western part of the large-scale low pressure system.

The structures of the cyclones are compared in the zonal-vertical sections through the cyclone centers in Fig. 5. The cyclone in ReT has typical TC structures including an upright trough axis, a warm core at the center, and active convection near the center (Fig. 5a). On the other hand, the cyclone in ReE has typical EC structures including westward tilt of the trough axis with increasing height and ascending motion of warm air in the eastern part (Fig. 5c). In $\mathrm{ReH}$ at $650 \mathrm{~h}$, the cyclone has a multiscale structure (Fig. 5b); the large-scale system has EC-like structures including tilted trough axis and ascending motion of warm air in the eastern part, whereas the small-scale convective core near the cyclone center shows TC-like structures including upright trough axis, a warm core, and intense convection.

The horizontal structure of the cyclone at $650 \mathrm{~h}$ in $\mathrm{ReH}$ are shown in Figs. 6a, 6d, and 6g. In the lower 
(a)

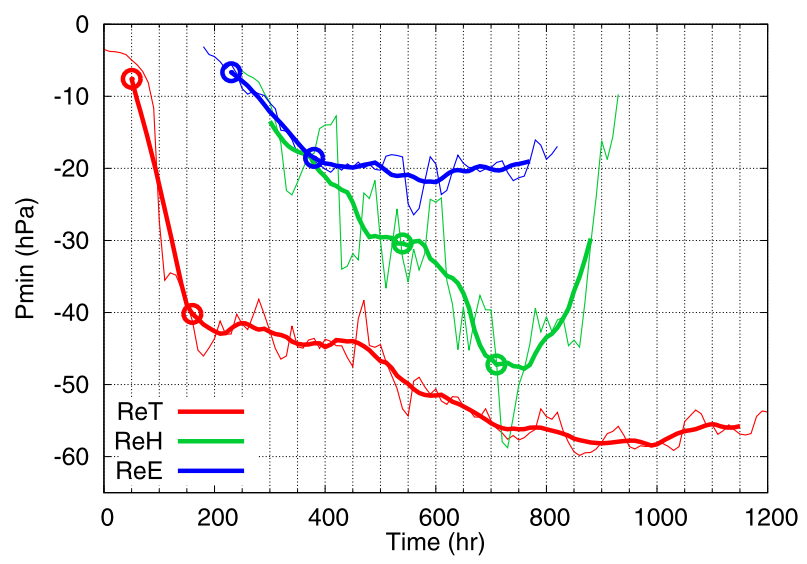

(c)

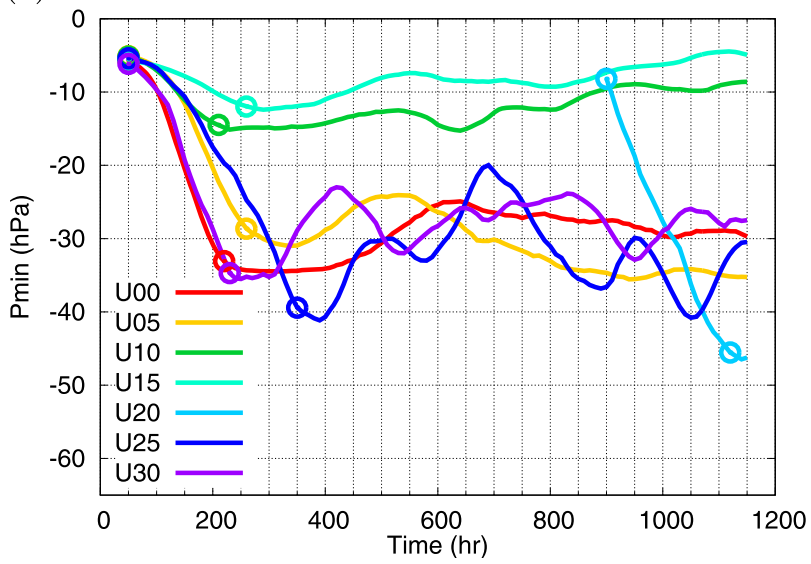

(b)

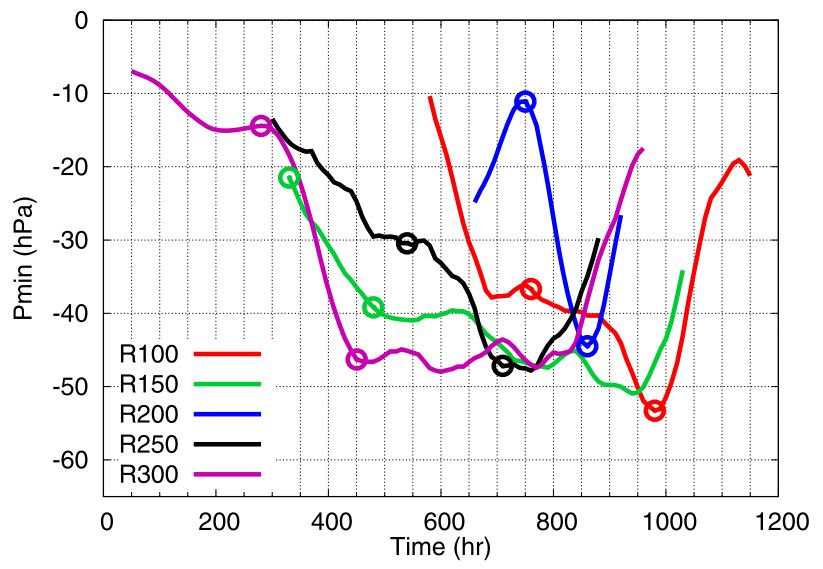

(d)

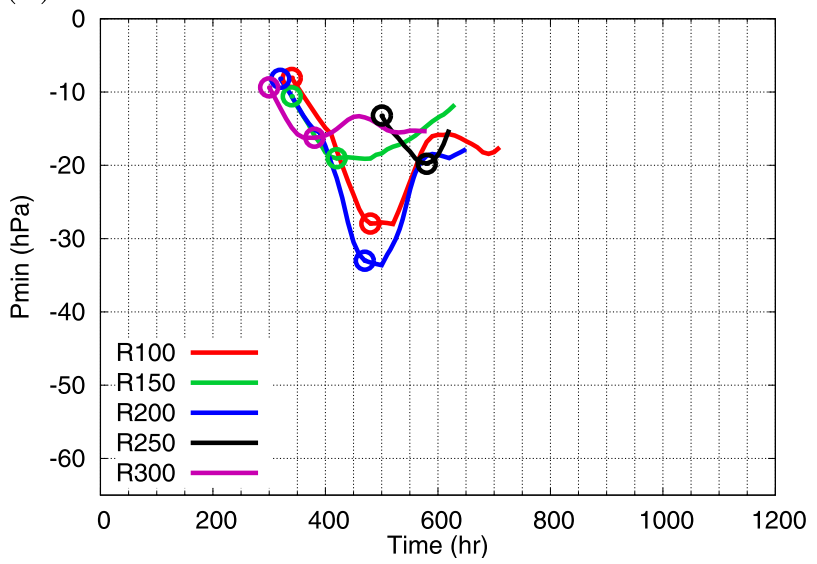

FIG. 3. Time evolution of the 100-h running mean of $P_{\min }$ (thick curves) for (a) the representative experiments, (b) ReH for different initial disturbance, (c) the shear experiments, and (d) $\mathrm{ReH}$ with no surface heat fluxes for different initial disturbances. The period between the two circles on each curve indicates the objectively detected development stage. Thin curves in (a) are the raw values of $P_{\text {min }}$.

troposphere (Fig. 6a), high equivalent potential temperature is advected from the south in the eastern part of the cyclone, which extends to the convective core at the cyclone center (see also Fig. 4i). This high equivalent potential temperature in the convective core is partly secluded by the air with low equivalent potential temperature originating from the northwest. In the upper troposphere (Fig. 6d), a large-scale cold trough is located to the west of the large-scale low pressure system in the lower troposphere, which corresponds to the westward tilt of the large-scale trough axis in Fig. 5b. The convective core is located under the eastern part of this large-scale cold trough in the upper troposphere. While the cold trough aloft is favorable for cumulus convection, the cold air is locally eroded around the convective core where large diabatic heating occurs (not shown). Around the upper-tropospheric trough, hook-shaped large potential vorticity extends from the northwest of the cyclone to the cyclone center (Fig. $6 \mathrm{~g}$ ).
In the lower troposphere, the cyclone center is accompanied by large potential vorticity. Near the cyclone center, intense convection occur on the west and north side of the center. These distributions of potential vorticity and convection resemble those of the tropical transition cases analyzed in Hulme and Martin (2009a,b), although we consider time-independent environmental fields.

\section{3) Sensitivity to Initial VORTEX}

To examine the sensitivity of the cyclones to the initial condition, the representative experiments are conducted by using different initial vortices, which have $r_{\max }$ of $100,150,200,250$, and $300 \mathrm{~km}$. The experiment with $r_{\max }$ of $x \mathrm{~km}$ is referred to as $\mathrm{R} x$, and the control experiment is equivalent to R250. As demonstrated in Yanase and Niino (2015), the maximum intensity of TCs and ECs are less sensitive to initial vortices (not shown). In the sensitivity experiments for HCs (Fig. 3b), cyclones develop 
(a) ReT

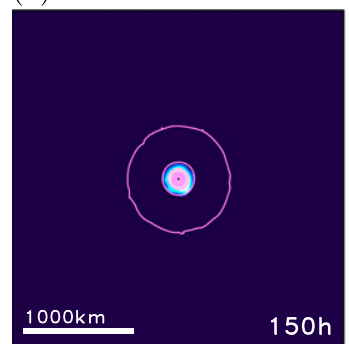

(e) $\mathrm{ReH}$

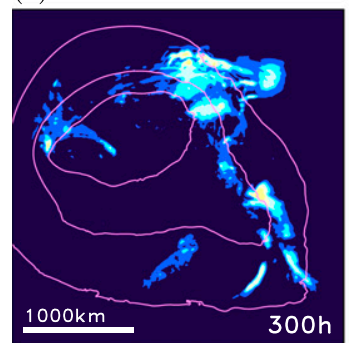

(i) $\mathrm{ReH}$

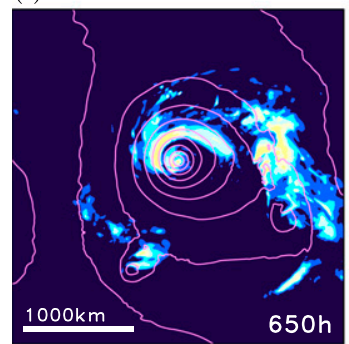

(b) ReT (magnified)

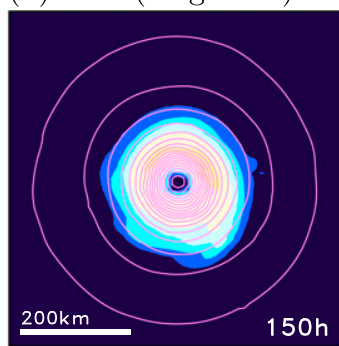

(f) $\mathrm{ReH}$

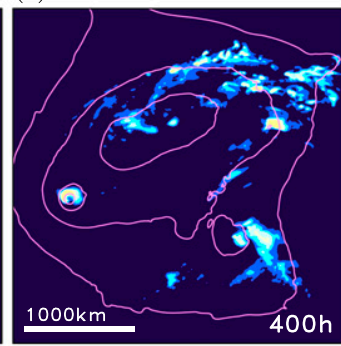

(j) $\mathrm{ReH}$

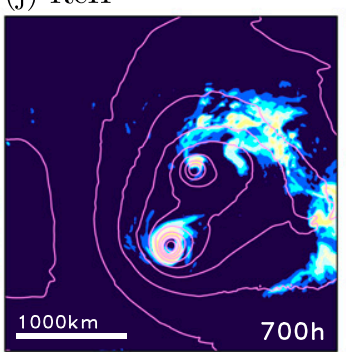

(c) $\operatorname{ReE}$

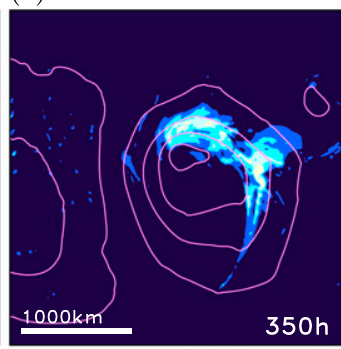

(g) $\mathrm{ReH}$

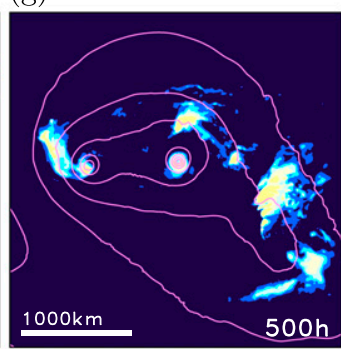

(k) $\mathrm{ReH}$

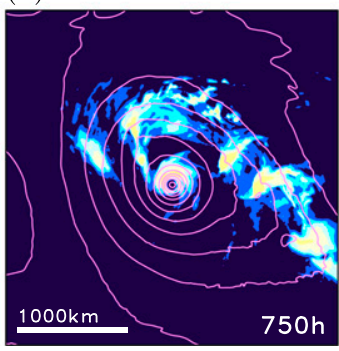

(d) T26U20F75

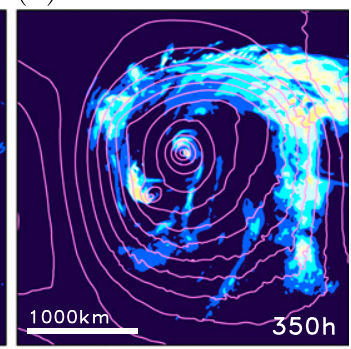

(h) $\mathrm{ReH}$

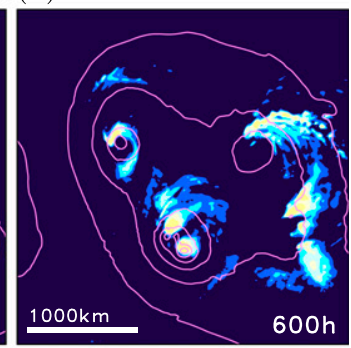

(l) $\mathrm{ReH}$

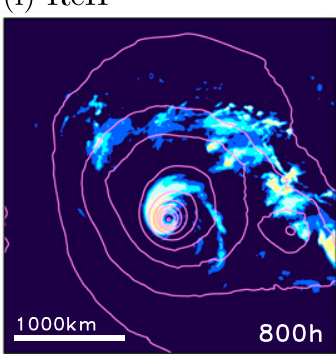

FIG. 4. Cloud pattern (shading) and SLP (purple contours) for (a) ReT at $150 \mathrm{~h}$, (b) magnified ReT at $150 \mathrm{~h}$, (c) ReE at $350 \mathrm{~h}$, (d) T26U20F75 at $350 \mathrm{~h}$, (e) ReH at $300 \mathrm{~h}$, (f) ReH at $400 \mathrm{~h}$, (g) ReH at $500 \mathrm{~h}$, (h) ReH at $600 \mathrm{~h}$, (i) $\mathrm{ReH}$ at $650 \mathrm{~h},(\mathrm{j}) \mathrm{ReH}$ at $700 \mathrm{~h},(\mathrm{k}) \mathrm{ReH}$ at $750 \mathrm{~h}$, and (l) $\mathrm{ReH}$ at $800 \mathrm{~h}$. The cloud pattern is represented by the column-integrated liquid and solid water. The contour interval is $5 \mathrm{hPa}$ for all the panels, except $2 \mathrm{hPa}$ for the magnified views. The panels show $3000 \mathrm{~km} \times 3000 \mathrm{~km}$ domains, in which the cyclone centers are placed at the center in the zonal direction, except that the magnified views show $600 \mathrm{~km} \times 600 \mathrm{~km}$ domains.

in all the experiments, whereas the timing and magnitude of the development vary among the experiments. The sensitivity of HC development to initial conditions was also shown in YN18, indicating that the prediction of HCs seems to be more difficult than those of TCs and ECs. Therefore, the present study focuses on general characteristics that are consistently observed in several experiments. (a) $\operatorname{ReT}(150 \mathrm{~h})$

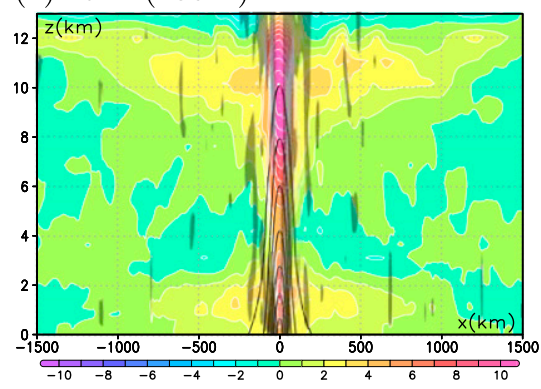

(b) $\mathrm{ReH}(650 \mathrm{~h})$

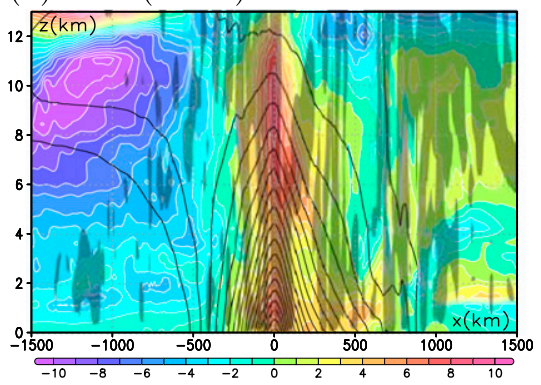

(c) $\operatorname{ReE}(350 \mathrm{~h})$

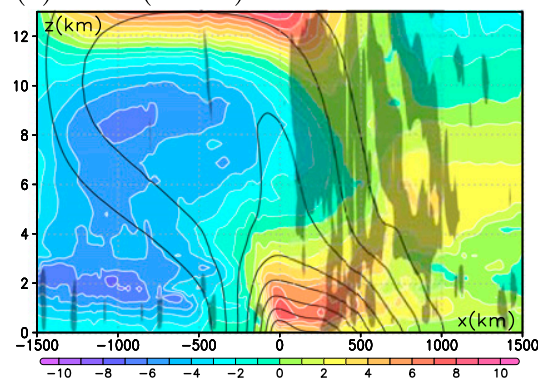

FIG. 5. Zonal-vertical sections through the cyclone centers at the development stages for (a) ReT at $150 \mathrm{~h}$, (b) ReH at $650 \mathrm{~h}$, and (c) ReE at $350 \mathrm{~h}$ showing the potential temperature anomaly (color shading; $\mathrm{K}$ ) and updrafts exceeding $2 \mathrm{~cm} \mathrm{~s}^{-1}$ (gray shading). Negative pressure anomaly by black contours at 5-hPa interval for (a) and at 2-hPa interval for (b) and (c). The cyclone centers are placed at the origins of the zonal coordinates. 
(a) $\operatorname{ReH}(650 \mathrm{~h})$

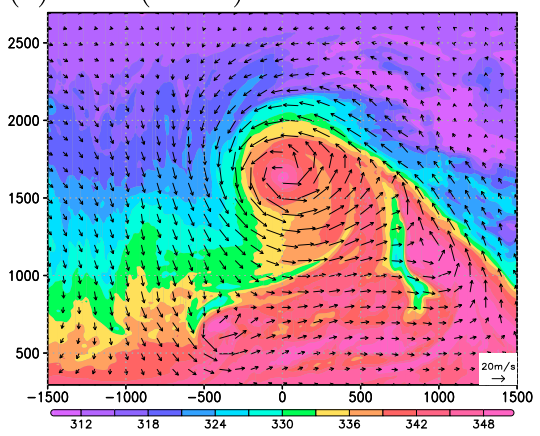

(d) $\operatorname{ReH}(650 \mathrm{~h})$

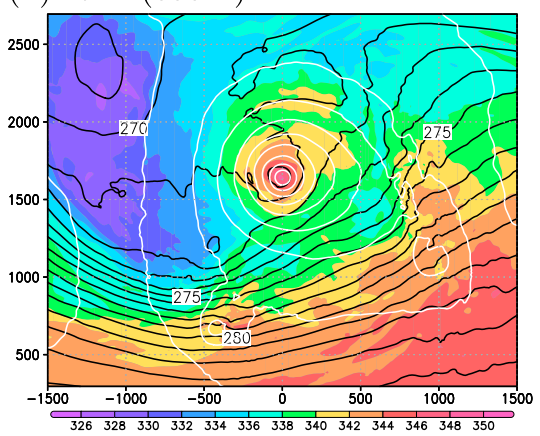

(g) $\mathrm{ReH}(650 \mathrm{~h})$

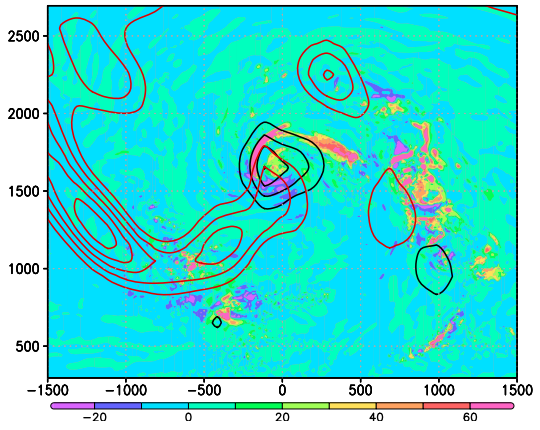

(b) T26U20F75 (350 h)

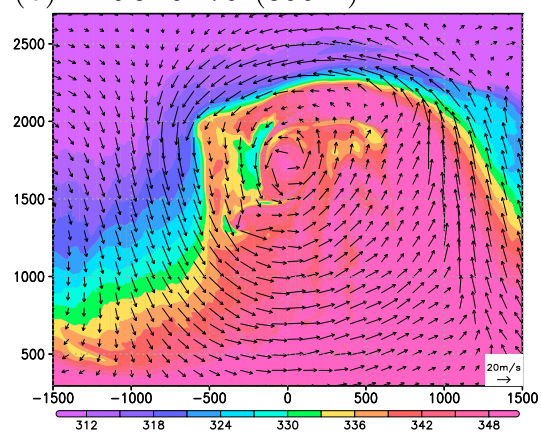

(e) T26U20F75 $(350 \mathrm{~h})$

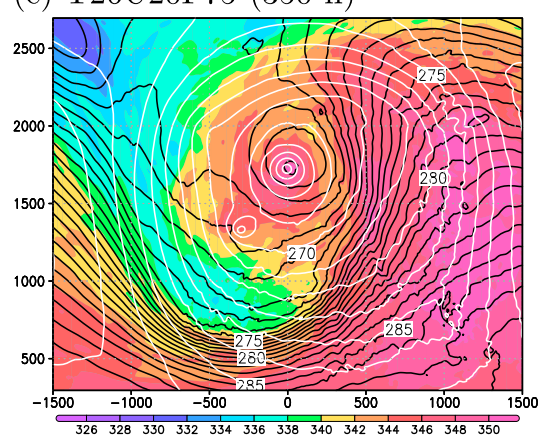

(h) T26U20F75 (350 h)

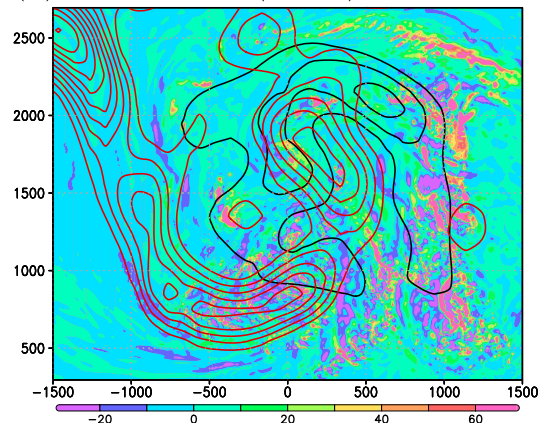

(c) $\operatorname{ShS}(250 \mathrm{~h})$

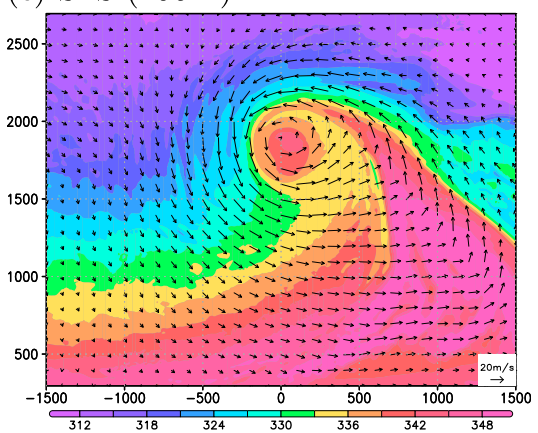

(f) $\mathrm{ShS}(250 \mathrm{~h})$

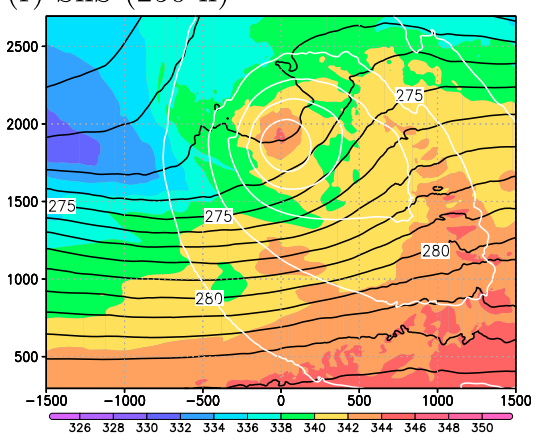

(i) $\mathrm{ShS}(250 \mathrm{~h})$

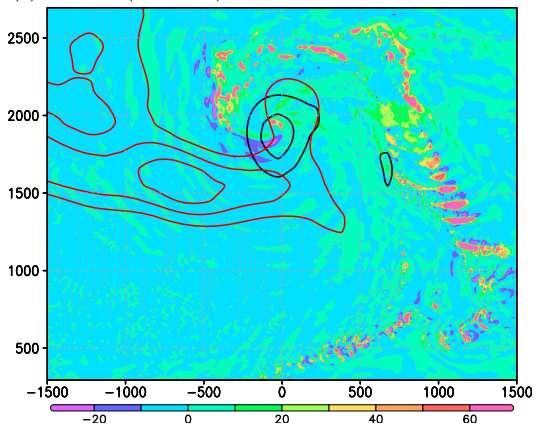

FIG. 6. Horizontal distribution of the cyclone structures for (a),(d),(g) ReH at $650 \mathrm{~h}$, (b),(e),(h) T26U20F75 at $350 \mathrm{~h}$, and (c),(f),(i) ShS at $250 \mathrm{~h}$. (a)-(c) Equivalent potential temperature (shading; K) and horizontal wind (arrows) at 1140-m height (850-900 hPa). (d)-(f) Potential temperature (shading; K) and pressure (black contours at 1-hPa interval) at 10140-m height (250-300 hPa), and SLP (white contours at 5-hPa interval). (g)-(i) Vertical wind (shading; $\mathrm{cm} \mathrm{s}^{-1}$ ) at 6140-m height (450-500 hPa), and potential vorticity greater than or equal to $1 \mathrm{PVU}$ (the filtered data; contours at 0.5 -PVU interval; $1 \mathrm{PVU}=10^{-6} \mathrm{~K} \mathrm{~kg}^{-1} \mathrm{~m}^{2} \mathrm{~s}^{-1}$ ) at 1140 -m height (black contours) and 10140-m height (red contours). The cyclone centers are placed at the origins of the zonal coordinates.

\section{4) Available potential energy budget}

The diabatic and baroclinic terms in the EPE budget analysis also shows the difference between a TC, an EC, and an HC (Fig. 7). In ReT, only the diabatic heating produces EPE. On the other hand, in ReE, the baroclinic term is larger than the diabatic term, particularly during its development stage (230-380 h). The EPE budget in $\mathrm{ReH}$ is intermediate between those in $\mathrm{ReT}$ and ReE; while the diabatic term is the largest as in ReT, the baroclinic term also produces EPE as in ReE. Thus, the ratio of diabatic and baroclinic terms is a useful index for comparing the mechanisms between the experiments, although there is a caveat that it is an index for the entire domain rather than an individual cyclone.

\section{b. Cyclones in a parameter space}

This subsection explores the cyclone development in the $T-U-F$ parameter space. Because cyclones tend to develop violently in the environment where all the three parameters are very large, the parameters are limited to the range that can marginally produce TCs, ECs, and 


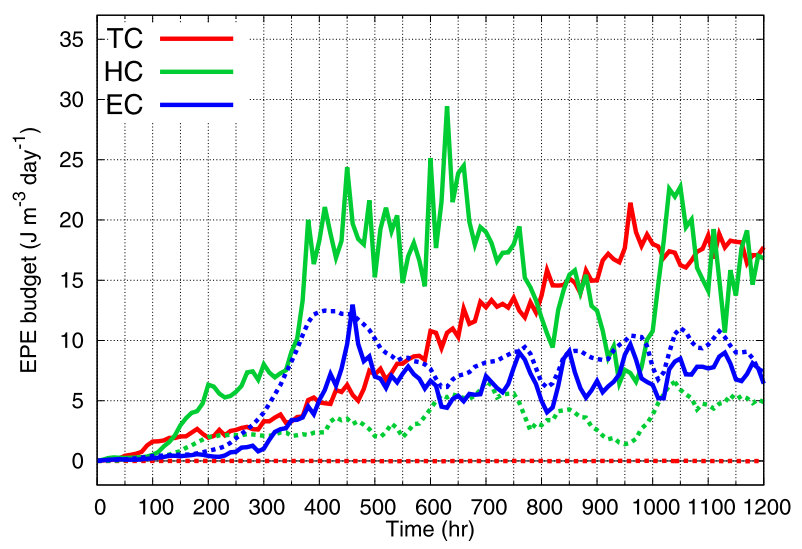

FIG. 7. Time evolution of diabatic term (solid curves) and baroclinic term (dashed curves) in the EPE budget for ReT (red), ReE (blue), and $\mathrm{ReH}$ (green).

HCs. The experiments examine the temperature at a $2^{\circ} \mathrm{C}$ interval between $18^{\circ}$ and $26^{\circ} \mathrm{C}$, the vertical shear at a $5 \mathrm{~m} \mathrm{~s}^{-1}$ interval between 0 and $20 \mathrm{~m} \mathrm{~s}^{-1}$, and the planetary vorticity at a $25 \%$ interval between $25 \%$ and $75 \%$.

The results of all the experiments in the $T-U-F$ space are summarized in Fig. 8a: the size of the circle indicates $-P_{\min }$ at the mature time, and the color indicates the ratio of the baroclinic term to the total generation terms in the EPE budget averaged for the development stage. In general, cyclones hardly develop in the region where all the parameters are small, whereas cyclones can develop in the region where the parameters are large. In the tropical environment (large $T$, small $U$, small $F$ ), the development of cyclones is dominantly attributed to diabatic process (the EPE ratio is smaller than 0.1 ) as in ReT. The structures of the cyclones in this region are also similar to those in ReT (not shown). On the opposite side, in the extratropical environment (small $T$, large $U$, large $F$ ), the development of cyclones is largely attributed to baroclinic process (the EPE ratio is larger than 0.5) as in ReE. The structures of the cyclones are also similar to those in $\operatorname{ReE}$ (not shown). In the environment with large $T$, large $U$, and large $F$, the development of cyclones are associated with the moderate EPE ratio (0.1-0.4) as in ReH. The cloud pattern and horizontal structure of a cyclone in T26U20F75 is shown in Figs. 4d and 6b, 6e, and 6h, respectively. The structures are qualitatively similar to those in ReH (T26U20F50; Figs. 4i and 6a,d,g); a convective core develops in a large-scale low pressure system with widespread cloud in the eastern part of the cyclone, and is accompanied by an upper-tropospheric cold trough with hook-shaped large potential vorticity to the west. Note that the environment of T26U20F75 is extreme compared to the real atmosphere.

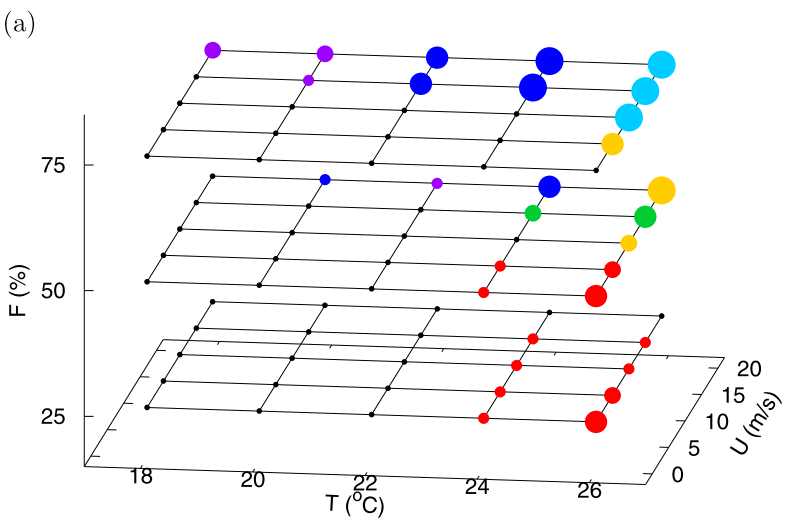

(b)

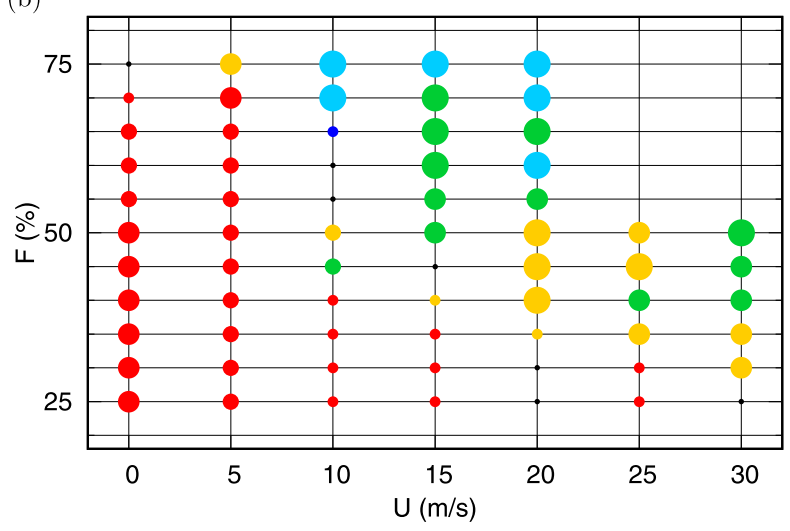

FIG. 8. The available potential energy budget and intensity for (a) the three-dimensional $T-U-F$ parameter space and (b) the twodimensional $U-F$ parameter space at $T=26^{\circ} \mathrm{C}$. The colors of the circles indicate the ratio of the baroclinic term to the total generation terms (the sum of the baroclinic and diabatic terms) averaged for the development stage. The sizes of the circles indicate $-P_{\min }$ at the mature time.

The relationship between ECs and HCs is observed along the $T$ axis at large $U$ and large $F$ (e.g., see the experiments from T18U20F75 to T26U20F75). With increasing the environmental temperature, the cyclones become more intense and more diabatic. This is consistent with previous studies in that diabatic heating intensifies baroclinic waves (Mak 1982; Wang and Barcilon 1986; Lambaerts et al. 2012; Booth et al. 2013). From this perspective, HCs may be considered as ECs that are intensified by diabatic heating; the present study roughly refers to the cyclones with the moderate EPE ratio as HCs. Although Fig. 8a shows no clear separation between ECs and HCs, it should be emphasized that HCs are remarkably associated with 
multiscale dynamics with TC-like convective cores as shown in ReH and T26U20F75.

The relationship between TCs and HCs is not straightforward. The $U-F$ parameter space at $T=26^{\circ} \mathrm{C}$ is explored in more detail (Fig. 8b): the experiments examine the planetary vorticity at $5 \%$ interval between $25 \%$ and $75 \%$, and extends the range of the vertical shear to $30 \mathrm{~m} \mathrm{~s}^{-1}$ for planetary vorticity $\leq 50 \%$. Along the $U$ axis at small $F$ (e.g., 25\%), cyclones become weaker with increasing the vertical shear. This is consistent with previous studies in that the vertical shear suppresses the TC development. However, at larger $F$ (e.g., $40 \%)$, cyclones develop for large $U\left(20 \mathrm{~m} \mathrm{~s}^{-1}\right.$ or more), whereas cyclones are still suppressed for moderate $U\left(10-15 \mathrm{~m} \mathrm{~s}^{-1}\right)$. Based on the EPE budget analysis, these cyclones for large $U$ seem to be HCs similar to $\mathrm{ReH}$ and T26U20F75 (Figs. 4d-1). As will be shown later, the cyclone in T26U25F40 also has characteristics of an $\mathrm{HC}$ with a TC-like convective core. In the $U-F$ space, HCs develop in the environment with the large baroclinicity $(U \times F)$, which is similar to the Eady growth rate for ECs (Lindzen and Farrell 1980; Hoskins and Valdes 1990) except for the influence of diabatic processes. Therefore, the unfavorable environment with moderate $U$ in which neither TCs nor HCs develop becomes narrower with increasing $F$, and finally disappears for $F$ exceeding $70 \%$. Thus, the environment favorable for cyclone development becomes continuous for large $F$.

\section{c. Asymmetric structures due to vertical shear}

The vertical shear plays several different roles in the cyclone dynamics as shown in Fig. 8b. As mentioned in section 1, Jones (1995) discussed two different mechanisms that cause asymmetric structures of cyclones: the warm-air-advection and downshear-tilt mechanisms. This subsection compares the cyclone structures in the experiments with different vertical shear at $T=26^{\circ} \mathrm{C}$ and $F=40 \%$ (shear experiments).

Time evolution of $P_{\min }$ for the shear experiments are shown in Fig. 3c. The cyclones develop for weak shear $\left(0 \sim 5 \mathrm{~m} \mathrm{~s}^{-1}\right)$ and for strong shear $\left(20 \mathrm{~m} \mathrm{~s}^{-1}\right.$ or larger $)$, whereas the cyclones are suppressed for moderate shear $\left(10-15 \mathrm{~m} \mathrm{~s}^{-1}\right)$. The cyclones in the weak shear experiments have structures similar to TCs (not shown). Hereafter, we focus on the cyclone structures in T26U10F40 for the moderate shear (ShM; see also Table 2) and T26U25F40 for the strong shear (ShS).

For the ShM, the cyclone is as small as that in ReT, but has an asymmetric cloud pattern (Figs. 9a,b). The asymmetric structure is clearly seen in the plan view focusing on the cyclone center (Fig. 10a). The center of the low pressure system at $3140-\mathrm{m}$ height is located slightly to the east of that at the sea level; that is, the trough axis is tilted to the downshear (east) side of the westerly vertical shear. The downshear side is also characterized by an updraft and low potential temperature around the radius of $50 \mathrm{~km}$. The azimuthalvertical distribution at this radius further highlights the asymmetric structure (Fig. 10b). The negative anomaly of potential temperature in the lower troposphere on the east side is consistent with the hydrostatic balance with the downshear tilt of the trough axis. Jones (1995) proposed that the cold anomaly is caused by adiabatic cooling in the updraft on the downshear side. Therefore, the tilt of the vortex, cold anomaly, and updraft on the downshear side is all consistent with the downshear-tilt mechanism. The convection on the downshear (slightly downshear left) side is also observed in TCs affected by vertical shear in previous studies (Ueno 2008). In summary, the cyclone in ShM is considered to be a TC asymmetrized by the vertical shear.

For the ShS, the structure of the cyclone changes through its life cycle (Figs. 9c-h) as in ReH. By 200 h, the cyclone is accompanied by a large-scale comma-shaped cloud pattern (Fig. 9d). At $250 \mathrm{~h}$, a convective core starts to develop in the western part of the widespread cloud (Fig. 9e), which becomes similar to a TC with a cloudfree eye to the west of the widespread cloud after $300 \mathrm{~h}$ (Fig. 9f-h). Figures 6c, 6f, and 6i show the horizontal distribution of various quantities at three vertical levels at $250 \mathrm{~h}$. High equivalent potential temperature in the lower troposphere is associated with the convective core and widespread cloud in the eastern part (Fig. 6c), whereas a cold trough with hook-shaped large potential vorticity is located to the west of the low pressure system in the lower troposphere (Figs. 6f,i). As these structures in ShS resemble those in $\mathrm{ReH}$ and T26U20F75, they are considered to be typical characteristics of HCs in the present experiments.

\section{Symmetric and asymmetric characteristics in the parameter space}

The results of the representative experiments and the EPE budget in the $T-U-F$ parameter space in the previous section may be summarized as in Table 3 . Since it is difficult to show the cyclone structures for all the experiments, we will utilize simple indices that can discriminate different types of cyclones in the present section. Because one of the obvious differences between a TC and an EC is axisymmetry of the cyclone structure, we attempt to develop a couple of indices based on symmetric and asymmetric structures of cyclones.

First, we transform the Cartesian coordinate to the cylindrical coordinate, which has the origin at the cyclone 
(a) ShM

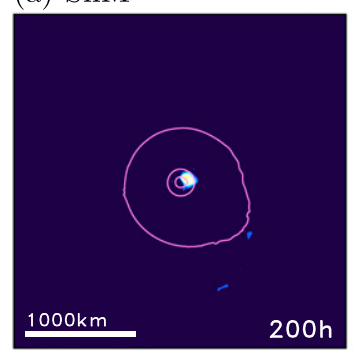

(e) ShS

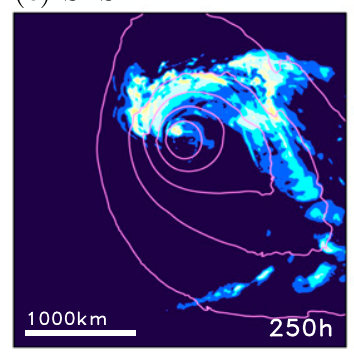

(b) ShM (magnified)

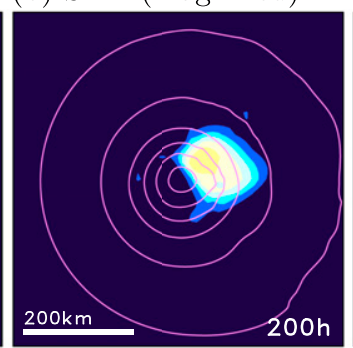

(f) $\mathrm{ShS}$ (c) ShS

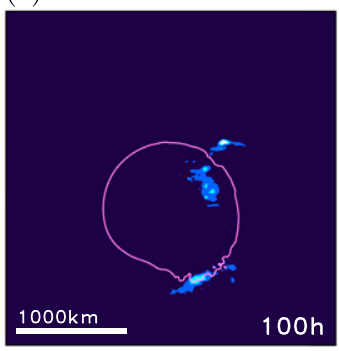

(g) $\mathrm{ShS}$ (d) $\mathrm{ShS}$

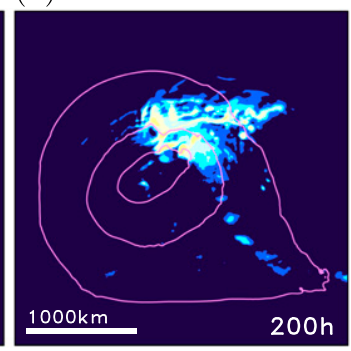

(h) ShS

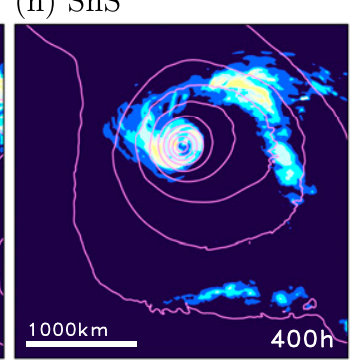

FIG. 9. As in Fig. 4, but for (a) ShM at 200 h, (b) magnified ShM at 200 h, (c) ShS at 100 h, (d) ShS at 200 h, (e) ShS at $250 \mathrm{~h}$, (f) ShS at $300 \mathrm{~h},(\mathrm{~g}) \mathrm{ShS}$ at $350 \mathrm{~h}$, and (h) ShS at $400 \mathrm{~h}$.

center. Symmetric structure is obtained by an azimuthal mean of a variable. Then "symmetric anomaly" at each radius is defined by the deviation from the value at the radius of $500 \mathrm{~km}$. "Asymmetric anomaly" is defined by the azimuthal wavenumber- 1 component. We utilize the wavenumber- 1 component whose amplitude is largest due east and west, which is associated with the asymmetric structures including the tilt of a trough axis and temperature advection as shown in Figs. 5 and 10. Note that the wavenumber- 1 component whose amplitude is largest due north and south would be mostly associated with the meridional temperature gradient of the environmental fields, and would be similar to the " $B$ " index of the cyclone phase space (Hart 2003) for eastward-moving cyclones in the present experiments. Thus, the present methodology effectively discriminates the cyclone structures from the environmental fields, whereas it is only available in case the direction of the horizontal temperature gradient of the environmental field is defined precisely.

Next, we normalize the magnitude of the anomalies by cyclone intensity to obtain a qualitative structure independent of the intensity. Here, the intensity is defined by the symmetric SLP anomaly that is averaged within the radius of $100 \mathrm{~km}$. Although the structures of cyclones can be represented by the potential temperature field as shown in Figs. 5a-c, we analyze the density field because it is directly related to SLP based on hydrostatic balance. The density field is normalized by the symmetric SLP anomaly (absolute value) divided by the gravitational acceleration, which gives a simple unit of inverse meters $\left(\mathrm{m}^{-1}\right)$.

The normalized symmetric density anomalies for the representative and shear experiments are shown by contours in Fig. 11. A negative anomaly (light density or warm air) in ReT is deep and concentrated near the cyclone center, which corresponds to a warm-core structure of a TC. On the other hand, the anomaly in $\mathrm{ReE}$ is shallow because of the tilt of the trough axis within an EC. In ShM, the anomaly is also shallow because the structure of a TC is modified by the vertical shear. In ReH and ShS, the HCs show deep structures. To assess the depth of the symmetric structure, we utilize the ratio of the symmetric density anomaly integrated between 6 and $8 \mathrm{~km}$ to that integrated between 0 and $2 \mathrm{~km}$, which is averaged over the radius between 0 and $100 \mathrm{~km}$ (regions Su and Sl, respectively, in Fig. 11f). Figure 12 overviews the $\mathrm{Su} / \mathrm{Sl}$ index for all the experiments in the $T-U-F$ space and the $U-F$ space at $T=26^{\circ} \mathrm{C}$ (see also Table 3). In general, the Su/S1 indices for typical TCs and for HCs are large (more than $\sim 0.3$ ), whereas those for asymmetric TCs and for typical ECs are small (less than $\sim 0.3$ ).

The normalized asymmetric density anomalies are shown by shading in Fig. 11, where the negative anomaly (orange and red) indicates that the air is lighter (warmer) in the eastern part of the cyclone than in the western part. Obviously, the cyclone in ReT has negligible asymmetry. In the other experiments, the asymmetric density anomaly is negative in the lower troposphere, because warm air is advected 


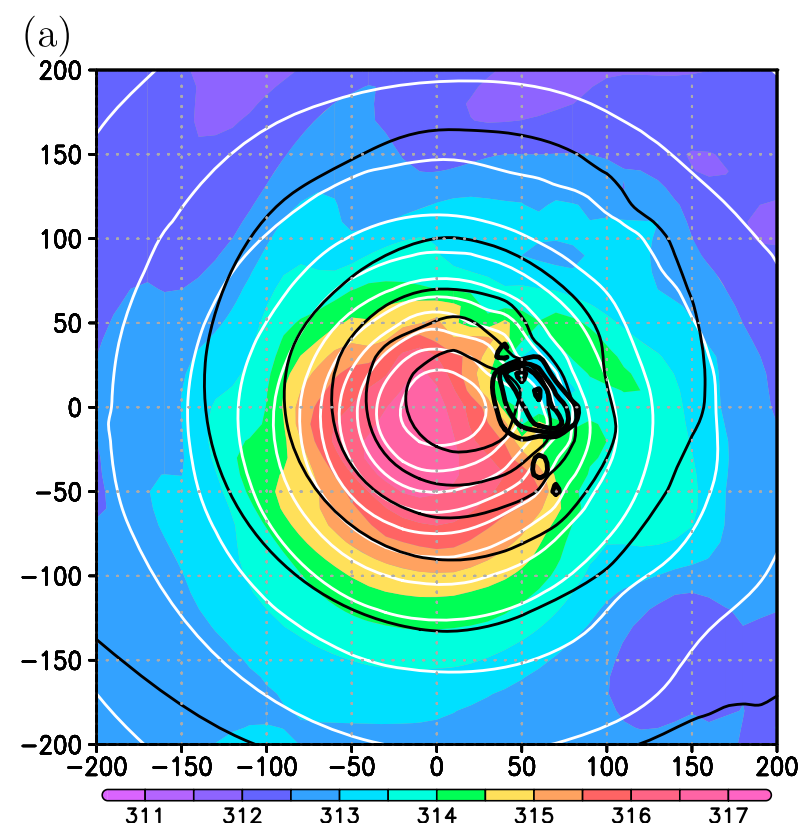

(b)

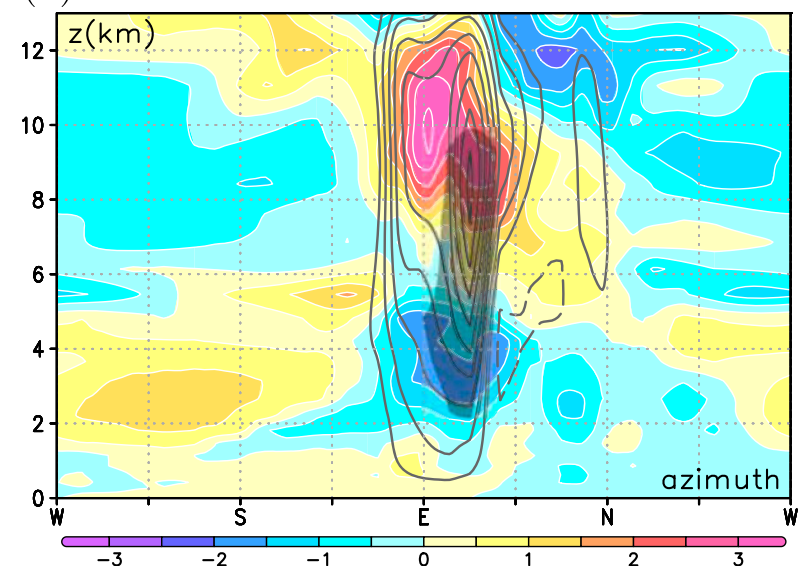

FIG. 10. Structure of the cyclone for ShM at $200 \mathrm{~h}$. (a) Horizontal distribution of potential temperature (shading; K), vertical wind (gray contours at $0.5 \mathrm{~m} \mathrm{~s}^{-1}$ interval), and pressure (black contours at $1-\mathrm{hPa}$ interval) at $3140-\mathrm{m}$ height $(650-700 \mathrm{hPa})$. SLP is also shown by white contours at $1-\mathrm{hPa}$ interval. The cyclone is placed at the origin of the horizontal coordinate. (b) Azimuthal-vertical distribution of the anomaly (difference from the azimuthal mean) of potential temperature (shading; K), vertical wind (gray contours at $0.5 \mathrm{~m} \mathrm{~s}^{-1}$ interval), and the condensational heating exceeding $30 \mathrm{~K} \mathrm{~h}^{-1}$ (gray shading) at the radius of $50 \mathrm{~km}$.

from the south on the east side of the cyclone. Only the exception is found near the cyclone center in ShM, where the air is dense (cold) between 2- and 4-km heights, as shown in Fig. 10. To assess these asymmetric structures, we utilize the asymmetric density anomalies multiplied by -1 that is integrated between 0 - and 2-km heights and averaged over the radius between 300 and $500 \mathrm{~km}$ [outer asymmetry index
TABLE 3. Cyclone types in the idealized experiments. From top to bottom the rows for each type describe (i) the environment, (ii) examples, (iii) intensity and EPE budget (see Fig. 8), and (iv) the structure.

\begin{tabular}{cc}
\hline \hline Type & Description \\
\hline
\end{tabular}

Typical TCs

(i) Large $T$, small $U$

(ii) ReT (T28U00F25; Figs. 4a,b, 5a), T26U00F40

(iii) Strong and diabatic development

(iv) A small and symmetric cyclone with an upright trough axis

Asymmetric TCs

(i) Large $T$, moderate $U$, small $F$

(ii) ShM (T26U10F40; Figs. 9a,b, 10), T26U10F30, T26U10F35

(iii) Weak and diabatic development (no development for relatively large $U$ : e.g., T26U20F25)

(iv) A small and asymmetric cyclone with a downshear-tilt trough axis

HCs

(i) Large $T$, large baroclinicity $(U \times F)$

(ii) $\mathrm{ReH}$ (T26U20F50; Figs. 4e-1, 5b, 6a,d,g), T26U20F75 (Figs. 4d, 6b,e,h), ShS (T26U25F40; Figs. 9c-h, 6c,f,i)

(iii) Strong and hybrid development

(iv) A multiscale and asymmetric cyclone with a TC-like convective core spawned within a baroclinic system

Typical ECs

No development

(i) Small $T$, large baroclinicity $(U \times \mathrm{F})$

(ii) ReE (T14U20F75; Figs. 4c, 5c), T18U20F75

(iii) Strong and baroclinic development

(iv) A large and asymmetric cyclone with an upshear-tilt trough axis

(i) Small $T$, small baroclinicity $(U \times F)$

(ii) T18U00F25, T22U20F25, T22U00F75

(iii) -

(iv) -

(Ao)], and that is integrated between 2- and 4-km heights and averaged over the radius between 0 and $100 \mathrm{~km}$ [inner asymmetry index (Ai)] as shown in Fig. 11f. Figure 13 overviews the Ao indices for all the experiments. In general, the Ao indices for typical TCs are small (less than 0.04), whereas those for typical ECs and HCs are large (more than 0.06). The Ai indices (not shown) robustly confirm the cold anomalies on the downshear side of the asymmetric TCs in T26U10F30, T26U10F35, and T26U10F40, whereas they do not show systematic characteristics for the other types of cyclones.

In summary, the symmetric and asymmetric indices can roughly confirm the relationship between cyclone structures 
(a) ReT

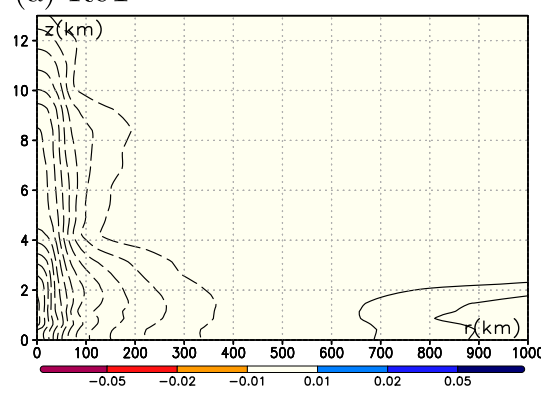

(d) ShM

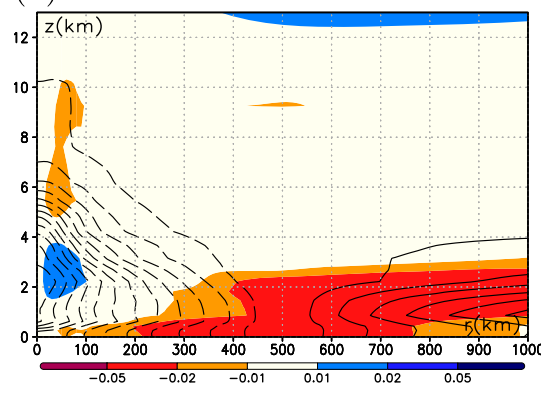

(b) $\mathrm{ReH}$

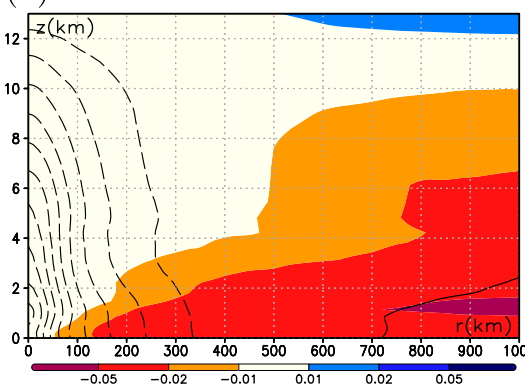

(e) $\mathrm{ShS}$

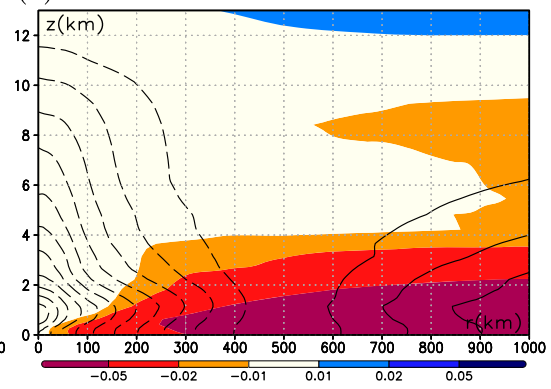

(c) $\operatorname{ReE}$

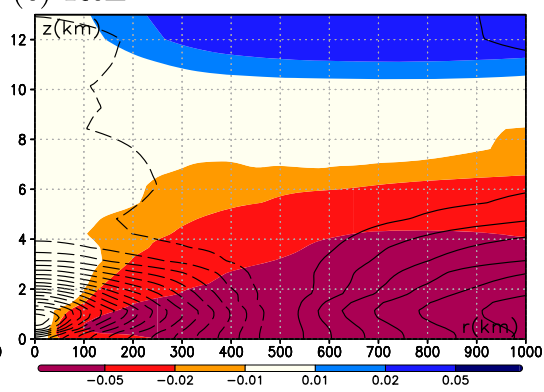

(f) Indices

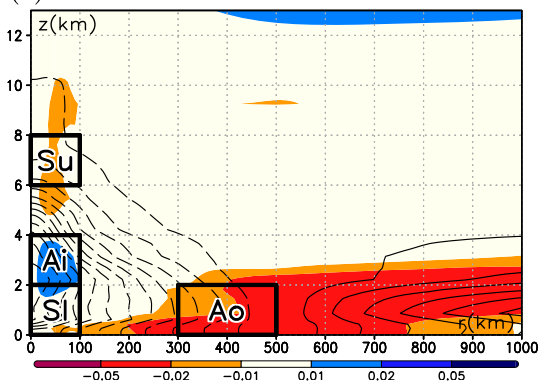

FIG. 11. Normalized symmetric and asymmetric structure averaged for the development stages of the cyclones for (a) ReT, (b) ReH, (c) ReE, (d) ShM, and (e) ShS. The symmetric density anomaly (dashed contours for negative values and solid contours for positive values at $0.02-\mathrm{km}^{-1}$ interval) and the asymmetric density anomaly (shading; $\mathrm{km}^{-1}$ ). A negative value of the asymmetric density anomaly (red colors) indicates that the air is light or warm on the east side of the cyclone center. (f) Regions used for the calculation of the indices.

and their environments, although the results exhibit fluctuations even in the same type of cyclones.

\section{Discussion}

\section{a. Spectrum of cyclones in the idealized and real atmosphere}

The present idealized experiments have demonstrated the close relationship between the environmental fields and cyclone dynamics systematically. In the real atmosphere, TCs and ECs are frequently observed in the tropics and extratropics, respectively, whereas cyclones are generally less frequent in the subtropics (Yanase et al. 2014). For example, the subtropical south Indian Ocean during the summer season is characterized by the environments with moderate values of $T, U$, and $F$ (Yanase and Niino 2015), which is not favorable for any types of cyclones as shown in Fig. 8a. However, HCs are active over particular subtropical oceans such as the North Atlantic Ocean in the autumn season (Guishard et al. 2009; McTaggart-Cowan et al. 2013), where the environments are characterized by large $T$, moderate or large $U$, and moderate $F$ (YN18) and are favorable for HCs as shown in Fig. 8b. Although the environment with large $T$, large $U$, and large $F$ is somewhat unrealistic in the present climate, it is useful for understanding what dynamics would operate in extreme environments possibly under a different climate.

Most of HCs in the present idealized experiments show multiscale dynamics. A large-scale baroclinic system has widespread cloud areas associated with northward advection of high equivalent potential temperature in the eastern part, and has an upper-tropospheric cold trough with hook-shaped large potential vorticity in the western part. This large-scale system spawns a TC-like convective core on the western part of the high equivalent potential temperature in the lower troposphere and under the eastern part of the upper-tropospheric cold trough. Such multiscale structures and dynamics are in fact observed in subtropical cyclones and tropical transition over the North Atlantic Ocean (Davis and Bosart 2004; Evans and Guishard 2009; Guishard et al. 2009; Hulme and Martin 2009a,b; Mauk and Hobgood 2012; Bentley and Metz 2016). It is also worth noting that the time evolution similar to tropical transition is partly reproduced even in the time-independent environmental fields.

Here, we discuss possible mechanisms for the development of a convective core in HCs. In the lower troposphere, the high equivalent potential temperature seems to be partly advected from the southeast (Figs. 6a-c), while it is secluded by the air with low equivalent potential temperature from the northwest at a later stage. Sea surface heat fluxes, particularly 
(a)

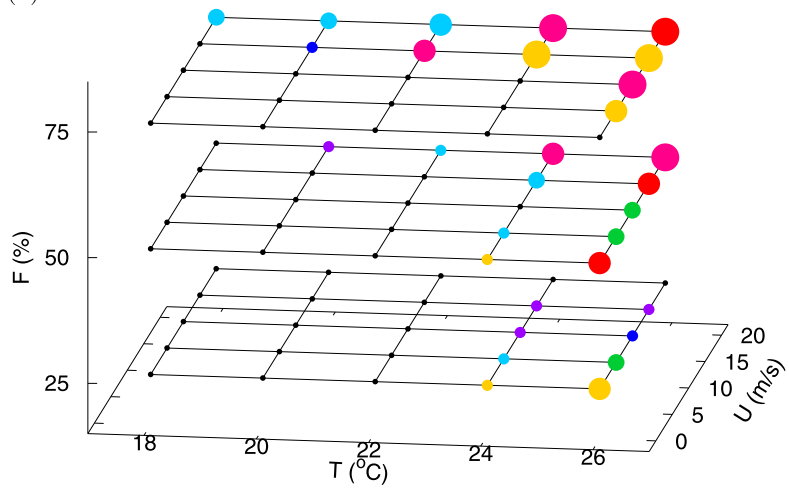

(b)

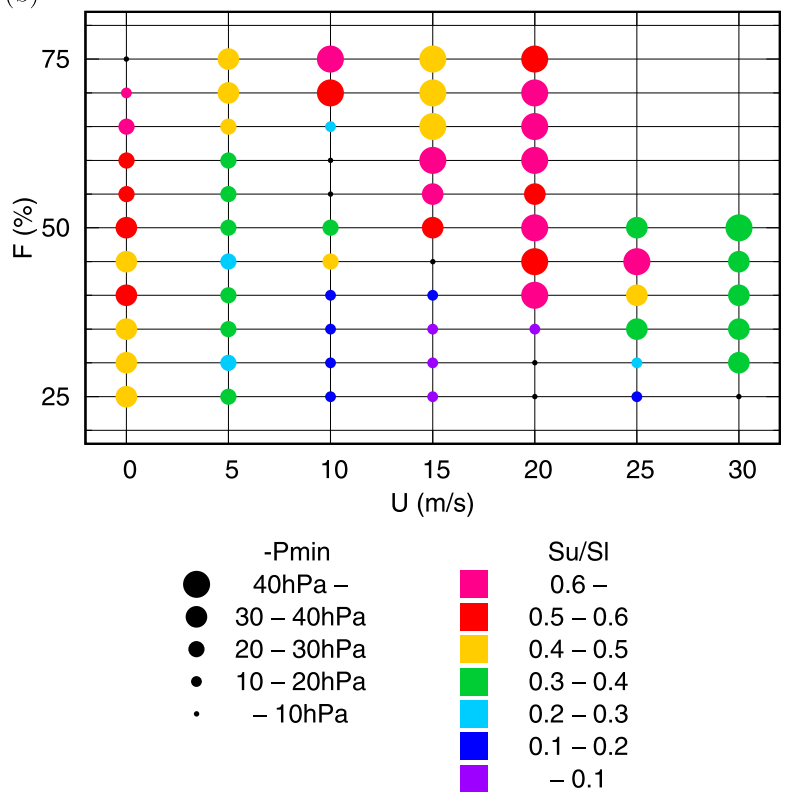

FIG. 12. The symmetry index $\mathrm{Su} / \mathrm{Sl}$ and intensity of the cyclones for (a) the three-dimensional $T-U-F$ parameter space and (b) the two-dimensional $U-F$ parameter space at $T=26^{\circ} \mathrm{C}$. The colors of the circles indicate the ratio of $\mathrm{Su}$ to $\mathrm{Sl}$ averaged for the development stage. The sizes of the circles indicate $-P_{\min }$ at the mature time.

latent heat flux, is also large because of the strong wind around the cyclone centers (not shown). The sensitivity experiments in which the surface heat fluxes are switched off in $\mathrm{ReH}$ resulted in the weaker development of cyclones (Figs. 3b,d), implying important roles of the surface heat fluxes. In the upper troposphere, the large-scale cold troughs may destabilize the troposphere and enhance convection (Figs. 6d-f). However, it is not easy to confirm large convective available potential energy in this region, because the upper troposphere is already warmed by diabatic heating. Davis (2010) also discussed the adiabatic upward forcing due to the upper-tropospheric trough. It should be also noted that the vertical shear in $\mathrm{ReH}$ is locally reduced around the cyclone center, which (a)

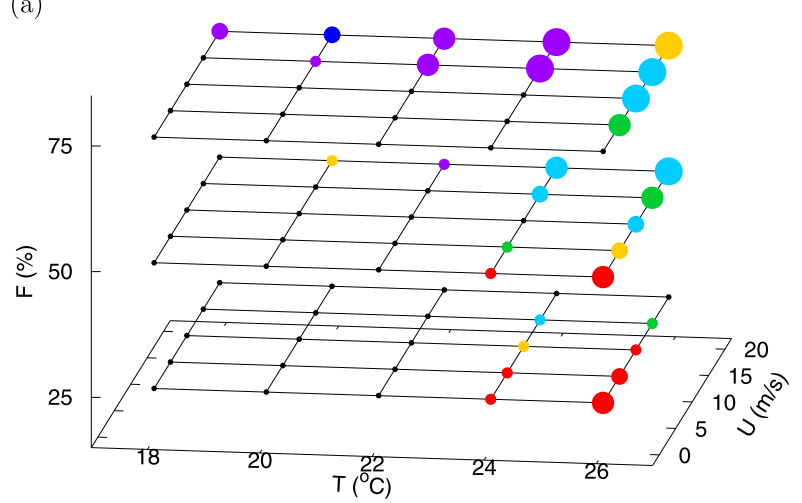

(b)

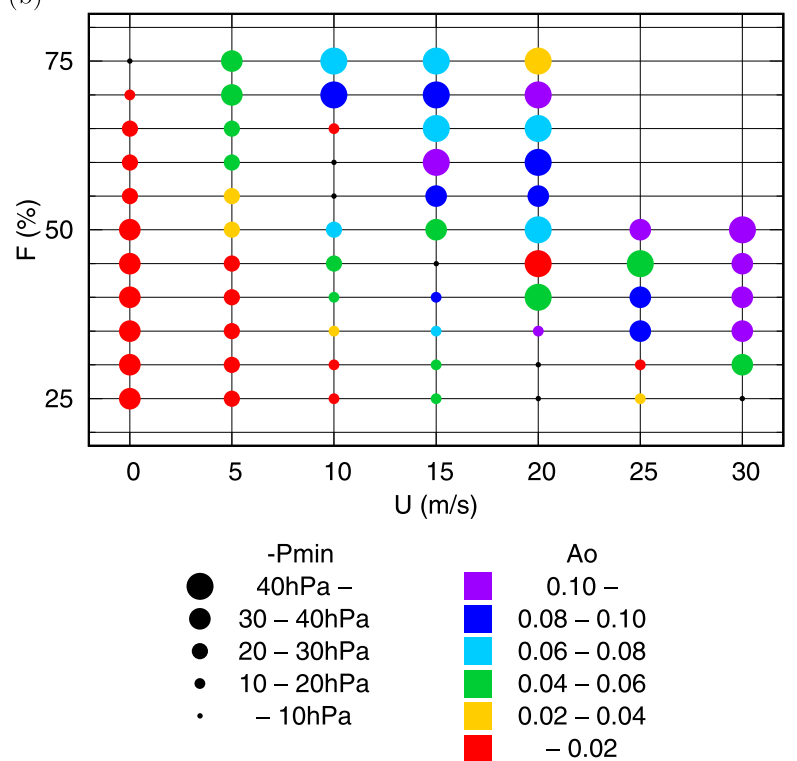

FIG. 13. The outer asymmetry index Ao and intensity of the cyclones for (a) the three-dimensional $T-U-F$ parameter space and (b) the two-dimensional $U-F$ parameter space at $T=26^{\circ} \mathrm{C}$. The colors of the circles indicate the Ao index averaged for the development stage. The sizes of the circles indicate $-P_{\min }$ at the mature time.

may be favorable for the development of the TC-like convective core (Davis and Bosart 2004; Hulme and Martin 2009a,b). The quantitative contributions of these dynamics for individual HCs should be clarified in more detail in future studies.

Another possible mechanism in moist baroclinic environments is a diabatic Rossby vortex (or wave), which propagates eastward through diabatic production of potential vorticity on the east side of the cyclone (Parker and Thorpe 1995; Moore and Montgomery 2004, 2005; Conzemius et al. 2007). A diabatic Rossby vortex has a shallow structure in the absence of the uppertropospheric forcing, which is different from baroclinic instability. In the present experiments, some cyclones have structures similar to diabatic Rossby vortices, although they are weak. For example, Fig. 14 
(a)

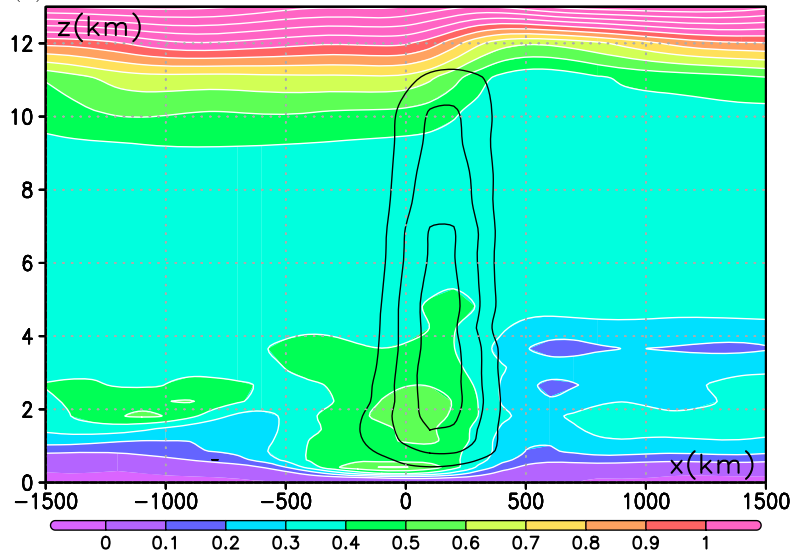

(b)

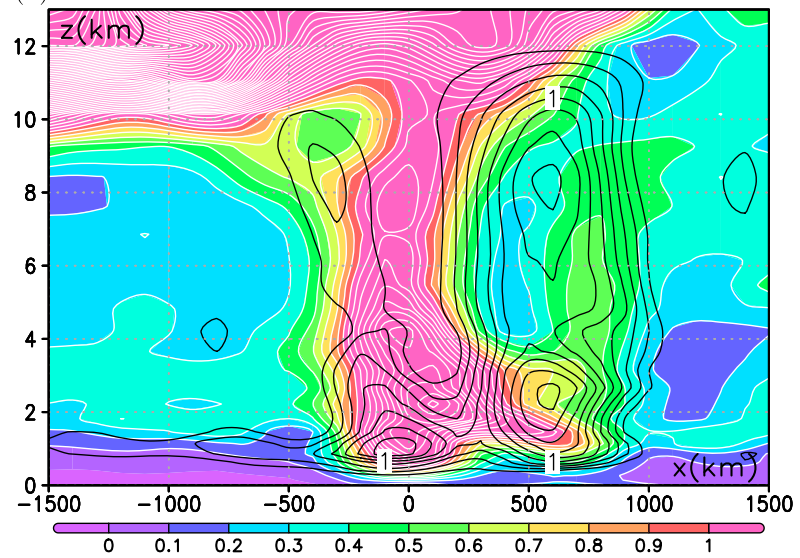

FIG. 14. Zonal-vertical sections through the cyclone centers for $\mathrm{ShS}$ at (a) 100 and (b) $250 \mathrm{~h}$ for the potential vorticity (shading; PVU) and positive condensational heating (contours at $0.2 \mathrm{~K} \mathrm{~h}^{-1}$ ). The filtered data are analyzed.

shows the vertical structure of the cyclone in ShS. During very early stages (Fig. 14a; see also Fig. 9c), the cyclone is characterized by a shallow structure of potential vorticity, little interaction with uppertropospheric potential vorticity, and condensational heating on the east side of the cyclone, all of which are consistent with a diabatic Rossby vortex. Because it is not easy to detect and classify them objectively based on their weak structures, we mainly focus on intense cyclones in the present study as a first step. During the development stage (Fig. 14b), the cyclone becomes deep and is accompanied by an upper-tropospheric trough with large potential vorticity (see also Figs. 6f,i). Because the upper-tropospheric potential vorticity seems to be associated with both diabatic production and meridional gradient of environmental potential vorticity, it is difficult to discriminate between a diabatic Rossby vortex and baroclinic instability.
It should be noted that the present idealized experiments are too simplified to understand effects of all the processes occurring in real cyclones. In the real atmosphere, the development and structures of cyclones may be affected by other environmental factors including large-scale vertical motion, humidity, static stability, horizontal shear, and the $\beta$ effect. Tropical and extratropical transition may occur in time-dependent environmental fields. In addition to the environmental fields, cyclones are also affected by transient external forcing including upper-tropospheric troughs. The relationship among these processes should also be examined systematically in future studies to understand the diversity in cyclones in the real atmosphere.

\section{b. Roles of vertical shear}

In the spectrum of cyclones, the vertical shear plays different roles in cyclone development depending on the cyclone types. For ECs, large vertical shear together with large planetary vorticity provide a favorable environment (Fig. 8a) because of the large baroclinicity or Eady growth rate (Lindzen and Farrell 1980; Hoskins and Valdes 1990). This characteristic is also observed for HCs in the $U-F$ space at $T=26^{\circ} \mathrm{C}$ (Fig. 8b). From this perspective, HCs in the present experiments may be regarded as baroclinic waves that are intensified by condensational heating. However, in contrast to normal mode disturbances in linear models, the HCs have multiscale structures where TC-like convective cores are embedded in large-scale baroclinic systems. For TCs, vertical shear suppresses the development of cyclones (Fig. 3c), which is consistent with previous studies (Frank and Ritchie 2001; Riemer et al. 2010; Finocchio et al. 2016). This suppressing effect separates the TCs for the weak shear from the HCs for the strong shear (Figs. 3c, 8b). Although the separation between TCs and HCs with respect to the vertical shear is wide at small planetary vorticity, it becomes narrower with increasing the planetary vorticity, and becomes ambiguous at high latitudes. This may be one of the explanations as to why the intensity of TCs at higher latitudes is less sensitive to vertical shear in the statistics in DeMaria (1996), in conjunction with DeMaria's explanation based on the Rossby penetration depth. It should be also noted that the separation between TCs and HCs with respect to the vertical shear becomes narrower with increasing the temperature as well (not shown).

The vertical shear also causes asymmetry of cyclone structure, which has an updraft and a cloud on the downshear side of the cyclone center. In the shear experiments (Fig. 9), the clouds on the downshear side are found for both moderate and strong shears (an 
asymmetric TC and an $\mathrm{HC}$ in Table 3, respectively), although the horizontal scale for the former is much smaller than that for the latter. As for the mechanisms discussed in Jones (1995), the structures of the cyclone for the moderate shear are consistent with the downshear-tilt mechanism (Fig. 10); the downshear side of the cyclone center is characterized by the updraft, cold anomaly, and tilt of the trough axis. On the other hand, the structures of the cyclone for the strong shear are consistent with the warm-air-advection mechanism (Figs. 6c,f,i); the downshear side is characterized by the updraft and the northward advection of high equivalent potential temperature. Note that the temperature anomalies in the lower troposphere are consistent with the hydrostatic balance with the tilt of the trough axes; the warm anomaly on the east side of the cyclone is associated with the westward tilt of the trough axis as shown in the large-scale structure in ECs and HCs, whereas the cold anomaly on the east side is associated with the eastward tilt as shown in ShM.

The zonal direction of the vortex tilt seems to be determined by at least two processes. The zonal advection merely tilts the vortex to downshear. When the vortex is affected by Earth's surface or tropopause, the Eady edge wave effect can tilt the vortex to upshear (Lackmann 2012). Although both processes are proportional to the magnitude of the vertical shear, the Eady edge wave effect plays more important role when the horizontal scale of the cyclone is larger than the Rossby radius of deformation. This is consistent with the results that the warm-airadvection mechanism in ShS occurs on much larger scale than the downshear-tilt mechanism in ShM.

\section{Summary}

The present idealized experiments explore the spectrum of cyclones from TCs to ECs in an environmental parameter space of temperature, vertical shear, and planetary vorticity. While the dynamics of individual types of cyclones have been investigated in previous studies, the parameter sweep experiments provide a systematic understanding of the relationship between cyclone dynamics and their environments in the same framework, which is particularly useful for intermediate environments where cyclone mechanisms are yet to be fully defined. HCs develop in the environments with high temperature and large baroclinicity as might be expected. While there is variability among the experiments, many HCs show a multiscale dynamics in which a large-scale baroclinic system spawns a small-scale TC-like convective core.
Such characteristics are also reported in previous observational studies on subtropical cyclones and tropical transition, although those in the real atmosphere form in a more complicated manner with different processes (Bentley et al. 2017; da Rocha et al. 2019).

The vertical shear plays two different roles in the asymmetric structures of cyclones. The shear experiments demonstrate that the downshear-tilt mechanism modifies small-scale structures of asymmetric TCs in the moderate shear environment, whereas the warm-airadvection mechanism generates large-scale ECs and HCs in the strong shear environment. This two-sided effect of the vertical shear makes the spectrum of cyclones less monotonic in the parameter space.

The present paper qualitatively overviews the different types of cyclones in different environments. The dynamics of HCs should be clarified in more detail using a high-resolution model that resolves cumulus convection. Ensemble simulations are also necessary for obtaining robust conclusions in the experiments for HCs that are sensitive to initial conditions. The present study only considers the environments based on the North Atlantic Ocean in autumn and minimal set of environmental factors that simulate synoptic-scale TCs, ECs, and HCs. More comprehensive and realistic experimental designs would be useful for understanding the diversity of synoptic- and mesoscale cyclones in the real atmosphere such as tropical/ extratropical transition, diabatic Rossby vortices, polar lows, and medicanes.

Acknowledgments. We acknowledge helpful comments by Drs. Eigo Tochimoto, Udai Shimada, Masaki Satoh, Izuru Takayabu, Kensuke Nakajima, Walter A. Robinson, and the three anonymous reviewers. The experiments was conducted using the Fujitsu PRIMEHPC FX100 in the Meteorological Research Institute. This research was partly supported by FLAGSHIP 2020, MEXT within Priority Study 4 (Advancement of Meteorological and Global Environmental Predictions Utilizing Observational "Big Data"), and by the Cooperative Program (No. 142, 2018) of Atmosphere and Ocean Research Institute, The University of Tokyo.

\section{REFERENCES}

American Meteorological Society, 2019: Subtropical cyclone. Glossary of Meteorology, http://glossary.ametsoc.org/wiki/ Subtropical_cyclone.

Beljaars, A., 1995: The parametrization of surface fluxes in largescale models under free convection. Quart. J. Roy. Meteor. Soc., 121, 255-270, https://doi.org/10.1002/qj.49712152203. 
Bentley, A. M., and N. D. Metz, 2016: Tropical transition of an unnamed, high-latitude, tropical cyclone over the eastern North Pacific. Mon. Wea. Rev., 144, 713-736, https://doi.org/ 10.1175/MWR-D-15-0213.1.

—_, D. Keyser, and L. F. Bosart, 2016: A dynamically based climatology of subtropical cyclones that undergo tropical transition in the North Atlantic basin. Mon. Wea. Rev., 144, 2049-2068, https://doi.org/10.1175/MWR-D-15-0251.1.

—_, L. F. Bosart, and D. Keyser, 2017: Upper-tropospheric precursors to the formation of subtropical cyclones that undergo tropical transition in the North Atlantic basin. Mon. Wea. Rev., 145, 503-520, https://doi.org/10.1175/ MWR-D-16-0263.1.

Booth, J. F., S. Wang, and L. Polvani, 2013: Midlatitude storms in a moister world: Lessons from idealized baroclinic life cycle experiments. Climate Dyn., 41, 787-802, https://doi.org/ 10.1007/s00382-012-1472-3.

Catto, J. L., 2016: Extratropical cyclone classification and its use in climate studies. Rev. Geophys., 54, 486-520, https://doi.org/ 10.1002/2016RG000519.

Charney, J. G., 1947: The dynamics of long waves in a baroclinic westerly current. J. Meteor., 4, 135-162, https://doi.org/ 10.1175/1520-0469(1947)004<0136:TDOLWI>2.0.CO;2.

— pression. J. Atmos. Sci., 21, 68-75, https://doi.org/10.1175/ 1520-0469(1964)021<0068:OTGOTH>2.0.CO;2.

Conzemius, R. J., R. W. Moore, and M. T. Montgomery, 2007: Mesoscale convective vortex formation in a weakly sheared moist neutral environment. J. Atmos. Sci., 64, 1443-1466, https://doi.org/10.1175/JAS3898.1.

da Rocha, R. P., M. S. Reboita, L. F. Gozzo, L. M. M. Dutra, and E. M. de Jesus, 2019: Subtropical cyclones over the oceanic basins: A review. Ann. N. Y. Acad. Sci., 1436, 138-156, https:// doi.org/10.1111/nyas.13927.

Davis, C. A., 2010: Simulations of subtropical cyclones in a baroclinic channel model. J. Atmos. Sci., 67, 2871-2892, https:// doi.org/10.1175/2010JAS3411.1.

— tropical transition of cyclones. Bull. Amer. Meteor. Soc., 85, 1657-1662, https://doi.org/10.1175/BAMS-85-11-1657.

DeMaria, M., 1996: The effect of vertical shear on tropical cyclone intensity change. J. Atmos. Sci., 53, 2076-2087, https://doi.org/ 10.1175/1520-0469(1996)053<2076:TEOVSO > 2.0.CO;2.

Deveson, A. C. L., and K. A. Browning, 2002: A classification of FASTEX cyclones using a height-attributable quasi-geostrophic vertical-motion diagnostic. Quart. J. Roy. Meteor. Soc., 128, 93-117, https://doi.org/10.1256/00359000260498806.

Eady, E. T., 1949: Long waves and cyclone waves. Tellus, 1 (3), 3352, https://doi.org/10.3402/tellusa.v1i3.8507.

Emanuel, K. A., 1986: An air-sea interaction theory for tropical cyclones. Part I: Steady-state maintenance. J. Atmos. Sci., 43, 585-604, https://doi.org/10.1175/1520-0469(1986)043<0585: AASITF $>2.0 . \mathrm{CO} ; 2$.

- , and R. Rotunno, 1989: Polar lows as Arctic hurricanes. Tellus, 41A, 1-17, https://doi.org/10.1111/j.1600-0870.1989.tb00362.x.

Evans, C., and Coauthors, 2017: The extratropical transition of tropical cyclones. Part I: Cyclone evolution and direct impacts. Mon. Wea. Rev., 145, 4317-4344, https://doi.org/10.1175/ MWR-D-17-0027.1.

Evans, J. L., and M. P. Guishard, 2009: Atlantic subtropical storms. Part I: Diagnostic criteria and composite analysis. Mon. Wea. Rev., 137, 2065-2080, https://doi.org/10.1175/ 2009MWR2468.1.
Finocchio, P. M., S. J. Majumdar, D. S. Nolan, and M. Iskandarani, 2016: Idealized tropical cyclone responses to the height and depth of environmental vertical wind shear. Mon. Wea. Rev., 144, 2155-2175, https://doi.org/ 10.1175/MWR-D-15-0320.1.

Fischer, M. S., B. H. Tang, and K. L. Corbosiero, 2017: Assessing the influence of upper-tropospheric troughs on tropical cyclone intensification rates after genesis. Mon. Wea. Rev., 145, 1295-1313, https://doi.org/10.1175/MWR-D-16-0275.1.

Frank, W., and E. Ritchie, 2001: Effects of vertical wind shear on the intensity and structure of numerically simulated hurricanes. Mon. Wea. Rev., 129, 2249-2269, https://doi.org/ 10.1175/1520-0493(2001)129<2249:EOVWSO > 2.0.CO;2.

Gray, W. M., 1975: Tropical cyclone genesis. Colorado State University Dept. of Atmospheric Sciences Paper 234, 121 pp.

Guishard, M., J. Evans, and R. Hart, 2009: Atlantic subtropical storms. Part II: Climatology. J. Climate, 22, 3574-3594, https:// doi.org/10.1175/2008JCLI2346.1.

Hart, R. E., 2003: A cyclone phase space derived from thermal wind and thermal asymmetry. Mon. Wea. Rev., 131, 585-616, https://doi.org/10.1175/1520-0493(2003)131<0585:ACPSDF> 2.0.CO;2.

— sition of Atlantic tropical cyclones. J. Climate, 14, 546-564, https:// doi.org/10.1175/1520-0442(2001)014<0546:ACOTET>2.0.CO;2.

Hendricks, E. A., M. T. Montgomery, and C. A. Davis, 2004: The role of "vortical" hot towers in the formation of Tropical Cyclone Diana (1984). J. Atmos. Sci., 61, 12091232, https://doi.org/10.1175/1520-0469(2004)061<1209: TROVHT $>2.0$.CO;2.

Hoskins, B. J., 1976: Baroclinic waves and frontogenesis part I: Introduction and Eady waves. Quart. J. Roy. Meteor. Soc., 102, 103-122, https://doi.org/10.1002/qj.49710243109.

— , and P. J. Valdes, 1990: On the existence of storm-tracks. J. Atmos. Sci., 47, 1854-1864, https://doi.org/10.1175/15200469(1990)047<1854:OTEOST>2.0.CO;2.

Hulme, A. L., and J. E. Martin, 2009a: Synoptic- and frontal-scale influences on tropical transition events in the Atlantic basin. Part I: A six-case survey. Mon. Wea. Rev., 137, 3605-3625, https://doi.org/10.1175/2009MWR2802.1.

$\longrightarrow$, and - 2009b: Synoptic- and frontal-scale influences on tropical transition events in the Atlantic basin. Part II: Tropical transition of Hurricane Karen. Mon. Wea. Rev., 137, 36263650, https://doi.org/10.1175/2009MWR2803.1.

Jones, S. C., 1995: The evolution of vortices in vertical shear. 1. Initially barotropic vortices. Quart. J. Roy. Meteor. Soc., 121, 821-851, https://doi.org/10.1002/qj.49712152406.

_ , and Coauthors, 2003: The extratropical transition of tropical cyclones: Forecast challenges, current understanding, and future directions. Wea. Forecasting, 18, 1052-1092, https://doi.org/ 10.1175/1520-0434(2003)018<1052:TETOTC $>2.0 . C O ; 2$.

Kain, J., and J. Fritsch, 1990: A one-dimensional entraining/detraining plume model and its application in convective parameterization. J. Atmos. Sci., 47, 2784-2802, https://doi.org/ 10.1175/1520-0469(1990)047<2784:AODEPM>2.0.CO;2.

Kitabatake, N., 2011: Climatology of extratropical transition of tropical cyclones in the western North Pacific defined by using cyclone phase space. J. Meteor. Soc. Japan, 89, 309-325, https://doi.org/10.2151/jmsj.2011-402.

Lackmann, G., 2012: Midlatitude Synoptic Meteorology: Dynamics, Analysis, and Forecasting. Amer. Meteor. Soc., 345 pp.

Lambaerts, J., G. Lapeyre, and V. Zeitlin, 2012: Moist versus dry baroclinic instability in a simplified two-layer atmospheric 
model with condensation and latent heat release. J. Atmos. Sci., 69, 1405-1426, https://doi.org/10.1175/JAS-D-11-0205.1.

Lapeyre, G., and I. M. Held, 2004: The role of moisture in the dynamics and energetics of turbulent baroclinic eddies. J. Atmos. Sci., 61, 1693-1710, https://doi.org/10.1175/15200469(2004)061<1693:TROMIT>2.0.CO;2.

Lin, Y., R. Farley, and H. Orville, 1983: Bulk parameterization of the snow field in a cloud model. J. Appl. Meteor., 22, 1065-1092, https:// doi.org/10.1175/1520-0450(1983)022<1065:BPOTSF>2.0.CO;2.

Lindzen, R. S., and B. Farrell, 1980: A simple approximate result for the maximum growth rate of baroclinic instabilities. J. Atmos. Sci., 37, 1648-1654, https://doi.org/ 10.1175/1520-0469(1980)037<1648:ASARFT>2.0.CO;2.

Mak, M., 1982: On moist quasi-geostrophic baroclinic instability. J. Atmos. Sci., 39, 2028-2037, https://doi.org/ 10.1175/1520-0469(1982)039<2028:OMQGBI>2.0.CO;2.

Mauk, R. G., and J. S. Hobgood, 2012: Tropical cyclone formation in environments with cool SST and high wind shear over the northeastern Atlantic Ocean. Wea. Forecasting, 27, 1433-1448, https://doi.org/10.1175/WAF-D-11-00048.1.

McTaggart-Cowan, R., T. J. Galarneau Jr., L. F. Bosart, W. M. Richard, and O. Martius, 2013: A global climatology of baroclinically influenced tropical cyclogenesis. Mon. Wea. Rev., 141, 1963-1989, https://doi.org/10.1175/MWR-D-12-00186.1.

Montgomery, M. T., and R. K. Smith, 2014: Paradigms for tropical cyclone intensification. Aust. Meteor. Oceanogr. J., 64, 37-66, https://doi.org/10.22499/2.6401.005.

Moore, R. W., and M. T. Montgomery, 2004: Reexamining the dynamics of short-scale, diabatic Rossby waves and their role in midlatitude moist cyclogenesis. J. Atmos. Sci., 61, 754-768, https:// doi.org/10.1175/1520-0469(2004)061<0754:RTDOSD>2.0.CO;2.

— , and - 2005: Analysis of an idealized, three-dimensional diabatic Rossby vortex: A coherent structure of the moist baroclinic atmosphere. J. Atmos. Sci., 62, 2703-2725, https:// doi.org/10.1175/JAS3472.1.

Mudrick, S. E., 1974: A numerical study of frontogenesis. J. Atmos. Sci., 31, 869-892, https://doi.org/10.1175/1520-0469(1974)031<0869: ANSOF $>2.0 . \mathrm{CO} ; 2$.

Murakami, M., 1990: Numerical modeling of dynamical and microphysical evolution of an isolated convective cloud-The 19 July 1981 CCOPE cloud. J. Meteor. Soc. Japan, 68, 107-128, https://doi.org/10.2151/jmsj1965.68.2_107.

Nakanishi, M., and H. Niino, 2004: An improved Mellor-Yamada level-3 model with condensation physics: Its design and verification. Bound.-Layer Meteor., 112, 1-31, https://doi.org/ 10.1023/B:BOUN.0000020164.04146.98.
Onogi, K., and Coauthors, 2007: The JRA-25 Reanalysis. J. Meteor. Soc. Japan, 85, 369-432, https://doi.org/10.2151/jmsj.85.369.

Ooyama, K., 1969: Numerical simulation of the life cycle of tropical cyclones. J. Atmos. Sci., 26, 3-40, https://doi.org/ 10.1175/1520-0469(1969)026<0003:NSOTLC > 2.0.CO;2.

Parker, D. J., and A. J. Thorpe, 1995: Conditional convective heating in a baroclinic atmosphere: A model of convective frontogenesis. J. Atmos. Sci., 52, 1699-1711, https://doi.org/ 10.1175/1520-0469(1995)052<1699:CCHIAB > 2.0.CO;2.

Reynolds, R. W., N. A. Rayner, T. M. Smith, D. C. Stokes, and W. Wang, 2002: An improved in situ and satellite SST analysis for climate. J. Climate, 15, 1609-1625, https://doi.org/10.1175/ 1520-0442(2002)015<1609:AIISAS > 2.0.CO;2.

Riemer, M., M. T. Montgomery, and M. E. Nicholls, 2010: A new paradigm for intensity modification of tropical cyclones: Thermodynamic impact of vertical wind shear on the inflow layer. Atmos. Chem. Phys., 10, 3163-3188, https://doi.org/ 10.5194/acp-10-3163-2010.

Saito, K., and Coauthors, 2006: The operational JMA nonhydrostatic mesoscale model. Mon. Wea. Rev., 134, 1266-1298, https://doi.org/10.1175/MWR3120.1.

Takayabu, I., 1986: Roles of the horizontal advection on the formation of surface fronts and on the occlusion of a cyclone developing in the baroclinic westerly jet. J. Meteor. Soc. Japan, 64, 329-345, https://doi.org/10.2151/jmsj1965.64.3_329.

Ueno, M., 2008: Effects of ambient vertical wind shear on the inner-core asymmetries and vertical tilt of a simulated tropical cyclone. J. Meteor. Soc. Japan, 86, 531-555, https://doi.org/ 10.2151/jmsj.86.531.

Wang, B., and A. Barcilon, 1986: Moist stability of a baroclinic zonal flow with conditionally unstable stratification. J. Atmos. Sci., 43, 705-719, https://doi.org/10.1175/1520-0469(1986)043<0705: MSOABZ $>2.0 . \mathrm{CO} ; 2$.

Yanase, W., and H. Niino, 2015: Idealized numerical experiments on cyclone development in the tropical, subtropical, and extratropical environments. J. Atmos. Sci., 72, 3699-3714, https:// doi.org/10.1175/JAS-D-15-0051.1.

—_, and —_, 2018: Environmental control of tropical, subtropical, and extratropical cyclone development over the North Atlantic Ocean: Idealized numerical experiments. Quart. J. Roy. Meteor. Soc., 144, 539-552, https://doi.org/ 10.1002/qj.3227.

- _ _ K. K. Iodges, and N. Kitabatake, 2014: Parameter spaces of environmental fields responsible for cyclone development from tropics to extratropics. J. Climate, 27, 652-671, https://doi.org/10.1175/JCLI-D-13-00153.1. 